\title{
A circRNA-miRNA-mRNA network identification for exploring underlying pathogenesis and therapy strategy of hepatocellular carcinoma
}

Dan-dan Xiong ${ }^{1 \dagger}$, Yi-wu Dang ${ }^{1 \dagger}$, Peng Lin², Dong-yue Wen ${ }^{2}$, Rong-quan He ${ }^{3}$, Dian-zhong Luo ${ }^{1}$, Zhen-bo Feng ${ }^{1 *}$ and Gang Chen ${ }^{1 *}$

\begin{abstract}
Background: Circular RNAs (circRNAs) have received increasing attention in human tumor research. However, there are still a large number of unknown circRNAs that need to be deciphered. The aim of this study is to unearth novel circRNAs as well as their action mechanisms in hepatocellular carcinoma (HCC).

Methods: A combinative strategy of big data mining, reverse transcription-quantitative polymerase chain reaction (RT-qPCR) and computational biology was employed to dig HCC-related circRNAs and to explore their potential action mechanisms. A connectivity map (CMap) analysis was conducted to identify potential therapeutic agents for HCC.

Results: Six differently expressed circRNAs were obtained from three Gene Expression Omnibus microarray datasets (GSE78520, GSE94508 and GSE97332) using the RobustRankAggreg method. Following the RT-qPCR corroboration, three circRNAs (hsa_circRNA_102166, hsa_circRNA_100291 and hsa_circRNA_104515) were selected for further analysis. miRNA response elements of the three circRNAs were predicted. Five circRNA-miRNA interactions including two circRNAs (hsa_circRNA_104515 and hsa_circRNA_100291) and five miRNAs (hsa-miR-1303, hsa-miR-142-5p, hsa-miR877-5p, hsa-miR-583 and hsa-miR-1276) were identified. Then, 1424 target genes of the above five miRNAs and 3278 differently expressed genes (DEGs) on HCC were collected. By intersecting the miRNA target genes and the DEGs, we acquired 172 overlapped genes. A protein-protein interaction network based on the 172 genes was established, with seven hubgenes (JUN, MYCN, AR, ESR1, FOXO1, IGF1 and CD34) determined from the network. The Gene Oncology, Kyoto Encyclopedia of Genes and Genomes and Reactome enrichment analyses revealed that the seven hubgenes were linked with some cancer-related biological functions and pathways. Additionally, three bioactive chemicals (decitabine, BW-B70C and gefitinib) based on the seven hubgenes were identified as therapeutic options for HCC by the CMap analysis.
\end{abstract}

Conclusions: Our study provides a novel insight into the pathogenesis and therapy of HCC from the circRNAmiRNA-mRNA network view.

Keywords: Hepatocellular carcinoma, CircRNA, ceRNA, CMap

\footnotetext{
*Correspondence: fengzhenbo_gxmu@163.com;

chengang@gxmu.edu.cn

${ }^{\dagger}$ Dan-dan Xiong and Yi-wu Dang contributed equally to this work

${ }^{1}$ Department of Pathology, First Affiliated Hospital of Guangxi Medical

University, 6 Shuangyong Road, Nanning 530021, Guangxi Zhuang

Autonomous Region, People's Republic of China

Full list of author information is available at the end of the article
}

(c) The Author(s) 2018. This article is distributed under the terms of the Creative Commons Attribution 4.0 International License (http://creativecommons.org/licenses/by/4.0/), which permits unrestricted use, distribution, and reproduction in any medium, provided you give appropriate credit to the original author(s) and the source, provide a link to the Creative Commons license, and indicate if changes were made. The Creative Commons Public Domain Dedication waiver (http://creativecommons.org/ publicdomain/zero/1.0/) applies to the data made available in this article, unless otherwise stated. 


\section{Background}

Circular RNA (circRNA), with a complete closed loop structure, is firstly identified in 1976 [1]. However, these transcripts without poly-A tail are ignored for a long time due to the limitation of traditional RNA detection methods. In recent years, with the development of high-throughput sequencing technology, a myriad of circRNAs has been found in the eukaryotic transcriptome [2]. With the features of cell-type specific [3] and highly conserved across species [4], circRNAs are thought to be new star RNAs which play important roles in various diseases, including human cancers $[5,6]$.

Competing endogenous RNAs (ceRNAs) are transcripts that act as miRNA sponges, modulating each other at post-transcriptional level via competely binding to shared miRNAs [7]. Recently, circRNAs have become new hotspots in ceRNA family since they have been demonstrated to harbor abundant conserved miRNA response elements (MREs) [8]. Increasing study has revealed that some circRNAs are involved in tumor initiation and progression by the ceRNA mechanism. For example, a classic cirRNA, ciRs7, is deemed to be a miRNA sponge, absorbing miR-7 and liberating the latter inhibitory effect on its target gene in many human cancers $[9,10]$. CircRNAs as ceRNAs mediating pathological processes has also been reported in HCC $[11,12]$. However, many unknown circRNAs still remain to be explored.

In this study, we employed a combinative strategy of gene chip and computational biology to investigate novel circRNAs and their potential action mechanisms in HCC. The flow chart recapitulating the present work is shown in Fig. 1 as follows: First, we collected HCCrelated microarray datasets providing expression profile of circRNAs from the Gene Expression Omnibus (GEO), obtaining differently expressed circRNAs (DECs) with RobustRankAggreg method and corroborating their expression using reverse transcription-quantitative polymerase chain reaction (RT-qPCR). To depict whether the DECs function as ceRNAs in HCC, we collected their sponge miRNAs and miRNA target genes, constructing a circRNA-miRNA-mRNA network. A protein-protein interaction (PPI) network was subsequently established and the hubgenes were identified. Then, Gene Oncology (GO), Kyoto Encyclopedia of Genes and Genomes (KEGG) and Reactome enrichment analyses on the hubgenes were performed to elucidate the potential pathogenesis of HCC. Furthermore, we conducted a connectivity map (CMap) analysis to acquire bioactive compounds for the treatment of $\mathrm{HCC}$, which provide a new insight into the latent therapeutic capacity of circRNAs in HCC.

\section{Methods}

\section{Screening of DECs in HCC from GEO}

Microarray datasets providing circRNA expression profile in HCC were achieved from the GEO database [13]. All raw expression data were normalized and $\log 2$-transformed. First, we used Limma, a Bioconductor package for differential analysis of microarray data, to determine DECs in each dataset with the criteria of $\mid \log 2$ (foldchange) $\mid>1$ and P-value $<0.05$ [14]. Then, we integrated and ranked all of the DECs with $R$ package RobustRankAggreg [15].

\section{Validation of DECs with RT-qPCR method}

Sixteen paired fresh frozen HCC tissues and corresponding adjacent non-tumor tissues were obtained from patients diagnosed with $\mathrm{HCC}$ at the First Affiliated Hospital of Guangxi Medical University (Nanning, Guangxi, People's Republic of China). No patient received any radiotherapy or chemotherapy before surgery. The Ethics Committee of the First Affiliated Hospital of Guangxi Medical University approved this study.

Total RNA was isolated with TRIzol ${ }^{\circledR}$ Reagent (Life technologies, Thermo Fisher Scientific, USA) following the manufacturer's instruction. Then, $1 \mu \mathrm{g}$ total RNA was reversed into $20 \mu \mathrm{l}$ complementary DNA (cDNA) with Geneseed $^{\circledR}$ II First Strand cDNA Synthesis Kit (Geneseed, Guangzhou, China). RT-qPCR was conducted using Geneseed $^{\circledR}$ qPCR SYBR ${ }^{\circledR}$ Green Master Mix (Geneseed) on ABI7500 system (Applied Biosystems, CA, USA) in line with the manufacturer's procedure. Beta-actin (Geneseed) was set as the endogenous reference. All of the primer sequences used in this study were synthesized by Geneseed and are displayed in Table 1. CircRNA expression was determined using the $2^{-\Delta C T}$ method. Significance between groups was analyzed by paired T-test with SPSS 22.0 (IBM, New York, USA). A P-value $<0.05$ denotes a statistical significance.

\section{Prediction of MREs}

The miRNA binding sites, also known as MREs, of those selected DECs, were predicted with two web tools, Cancer-Specific CircRNA (CSCD) [16] and Circular RNA Interactome (CircInteractome) [17]. We identified overlapped miRNAs of the two algorithms as potential target miRNAs of the DECs.

\section{Verification of miRNA expression based on data from GEO and TCGA}

Microarray datasets providing miRNA expression profile in HCC were obtained from the public databases GEO and The Cancer Genome Atlas (TCGA) [18]. The retrieval terms were as follows: (hepatocellular OR hepatic OR liver OR HCC) and ("cancer" OR "tumor" OR 


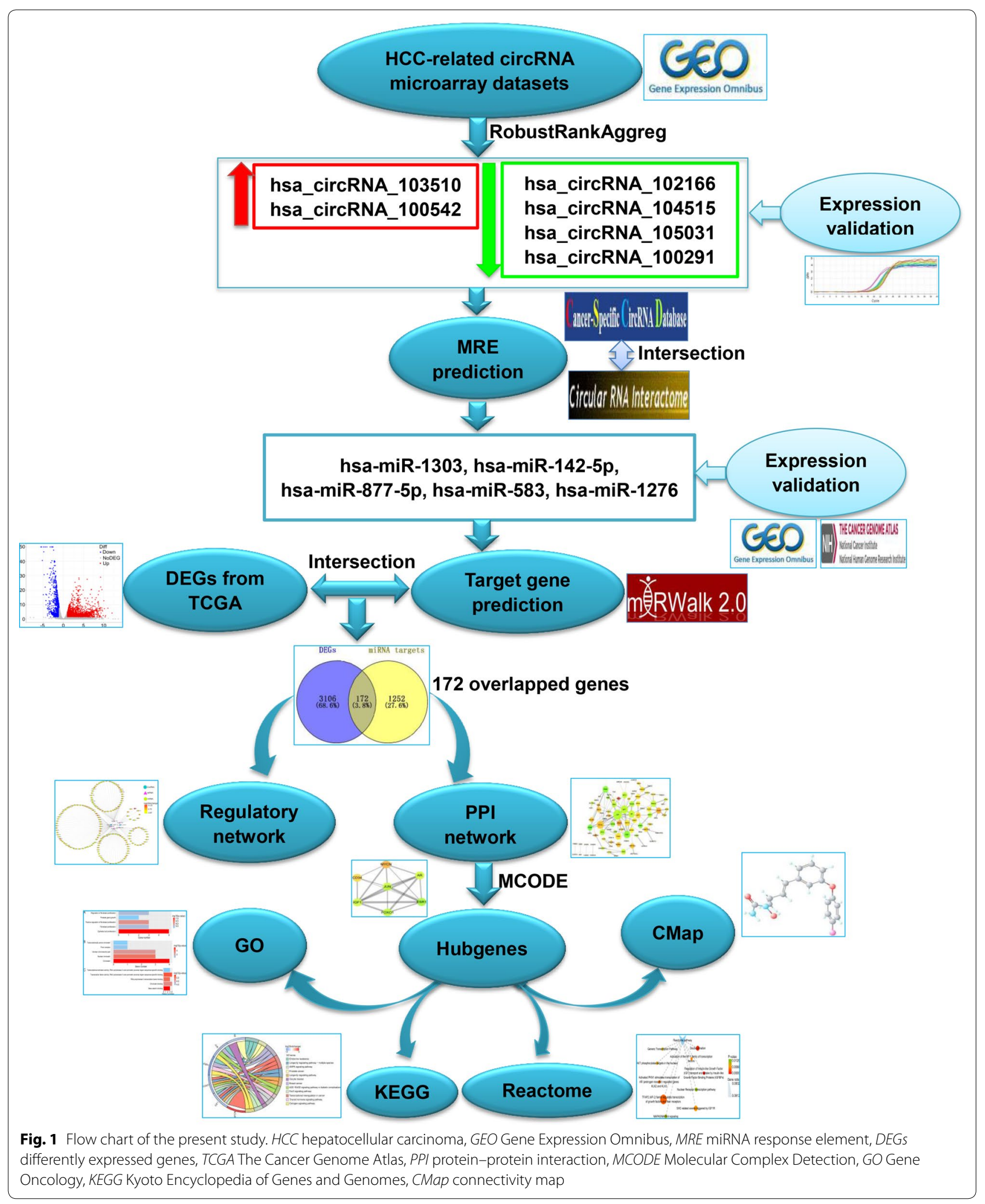


Table 1 Primer sequences for reverse transcription-quantitative polymerase chain reaction

\begin{tabular}{|c|c|c|c|}
\hline \multirow[t]{2}{*}{ Gene ID } & \multicolumn{2}{|l|}{ Primer sequence } & \multirow{2}{*}{$\begin{array}{l}\text { Product } \\
\text { length } \\
\text { (bp) }\end{array}$} \\
\hline & Forward $\left(5^{\prime}-3^{\prime}\right)$ & Reverse $\left(5^{\prime}-3^{\prime}\right)$ & \\
\hline ACTB & CATGTACGTTGCTATCCAGGC & CTCCTTAATGTCACGCACGAT & 250 \\
\hline hsa_circRNA_102166 & TACGTTGATCACCAAGGGCT & CTTCTGCTTTGGCTGTGACA & 126 \\
\hline hsa_circRNA_104515 & CTTTATAACTATAGGGTACTGG & GTCTCCTCTGGTTCATTG & 150 \\
\hline hsa_circRNA_105031 & ACTACAGGCAATCAGGGTTC & GTACAAGTTCTGCAGGAACGA & 118 \\
\hline hsa_circRNA_100291 & CATTCTTATAGTTGTAAGCTTAG & CATAGGAGAAAGCATCATTAT & 131 \\
\hline
\end{tabular}

ACTB beta-actin

"tumour" OR "carcinoma” OR "neoplasm" OR "malignan") and (miRNA OR microRNA OR miR OR "noncoding RNA" OR ncRNA OR "noncoding RNA" OR "non coding RNA").

We screened available datasets based on the listed inclusion criteria: (1) all of the patients were diagnosed with HCC; (2) the studies must contain circRNA expression data both in cancerous and normal liver tissues; and (3) the sample sizes in tumor and non-tumor group were at least three.

Two revivers extracted the basic information of each included record: miRNA type, first author and published year, region, data source, platform, number of case, and expression level of miRNA. Any divergences were settled via discussion with a third investigator.

The combined standard mean difference (SMD) and 95\% confidence interval (95\% CI) were computed by STATA 12.0 (StataCorp, College Station, TX, USA). A SMD $>0$ represents high expression of miRNA in HCC samples than in normal controls. The corresponding 95\% CI do not cross 1 and a P-value $<0.05$ suggest a statistical significance.

\section{Prediction of miRNA target genes}

The miRNA-mRNA interactions were predicted with miRWalk 2.0 [19], which involves 12 predicted algorithms (Targetscan, RNAhybrid, RNA22, PITA, Pictar2, miRWalk, Microt4, miRNAMap, miRDB, mirbridge, miRanda and miRMap). Target genes forecasted by at least eight algorithms were selected for further analysis.

\section{Collection of differently expressed genes (DEGs) of HCC form TCGA}

RNA-sequencing (RNA-seq) data containing 374 HCC samples and 50 normal controls was downloaded from the TCGA. DEGs were determined by the edgeR package [20] in Bioconductor with the filter criteria of $\mid \log 2$ (foldchange) $\mid>1$ and adjust $\mathrm{P}$-value $<0.05$.

\section{Construction of circRNA-miRNA-mRNA network}

The overlapping genes between the predicted miRNA target genes and the DEGs were obtained for circRNAmiRNA-mRNA network construction. The Cytoscape 3.6.1 software [21] was used to visualize the regulatory network.

\section{Establishment of PPI network and identification of hub-genes}

A PPI network was established by the STRING (v10.5) [27924014] and visualized by the Cytoscape 3.6.1. Then, the "Molecular Complex Detection" (MCODE), a clustering algorithm identifying locally densely connected regions in a large PPI network based on a node-weighting arithmetic [22], was employed to recognize highly interacted hubgene clustering.

\section{GO, KEGG and Reactome enrichment analyses}

GO annotation and KEGG pathway analyses were conducted by clusterProfiler, an $\mathrm{R}$ package for functional classification and enrichment of gene clusters using hypergeometric distribution [23]. Reactome pathway analysis was performed by Reactome FI, a plugin of Cytoscape for network and pathway analysis [24].

Table 2 Basic information of the three microarray datasets from GEO

\begin{tabular}{|c|c|c|c|c|c|c|}
\hline Data source & Platform & First author & Year & Region & Sample size (T/N) & $\begin{array}{l}\text { Number } \\
\text { of circRNAs }\end{array}$ \\
\hline GSE78520 & GPL19978 & $\mathrm{LiC}$ & 2016 & China & $3 / 3$ & 4451 \\
\hline GSE94508 & GPL19978 & FuL & 2017 & China & $5 / 5$ & 2572 \\
\hline GSE97332 & GPL19978 & Han D & 2017 & China & $7 / 7$ & 3471 \\
\hline
\end{tabular}




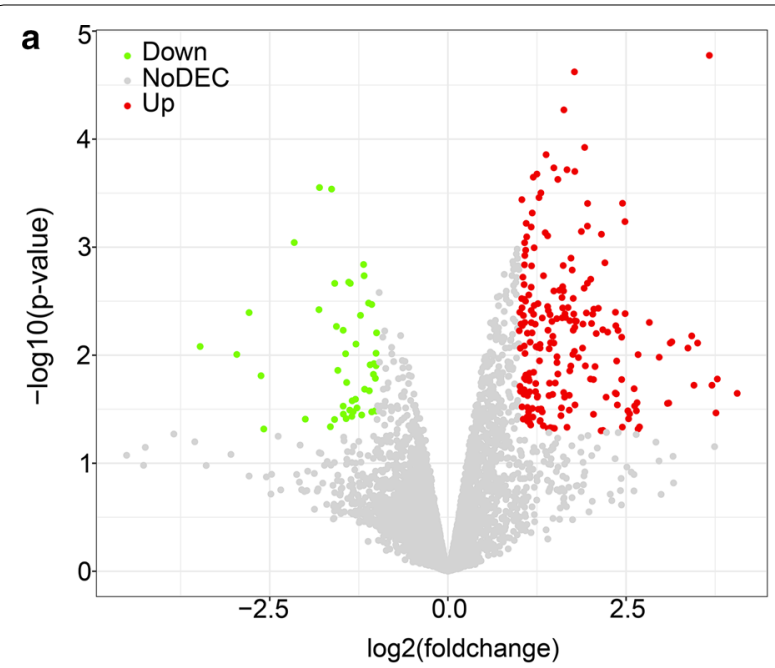

b
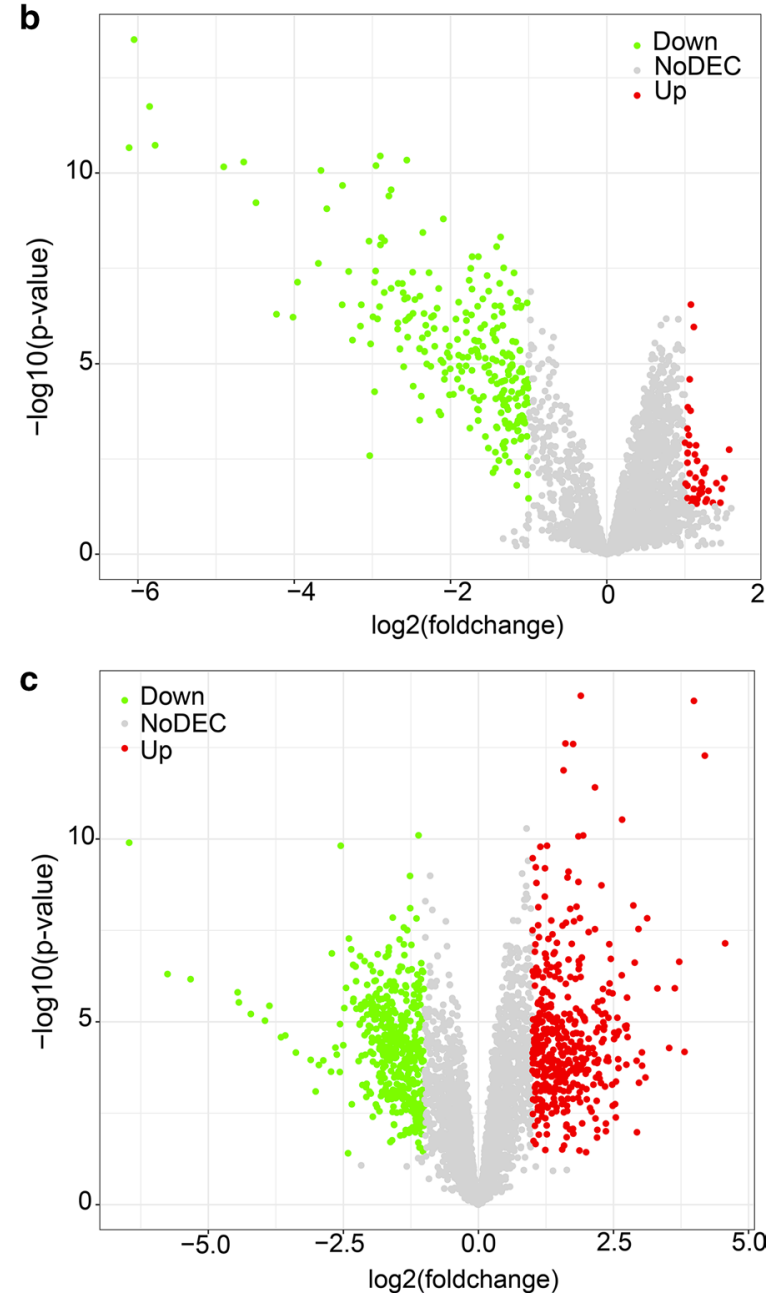

Fig. 2 Volcano plots for DECs in HCC based on the three microarray datasets from GEO: a GSE78520, b GSE94508, c GSE97332. The volcano plot was generated by $R$ package 'ggplot2. DECs differently expressed circRNAs, HCC hepatocellular carcinoma, GEO Gene Expression Omnibus

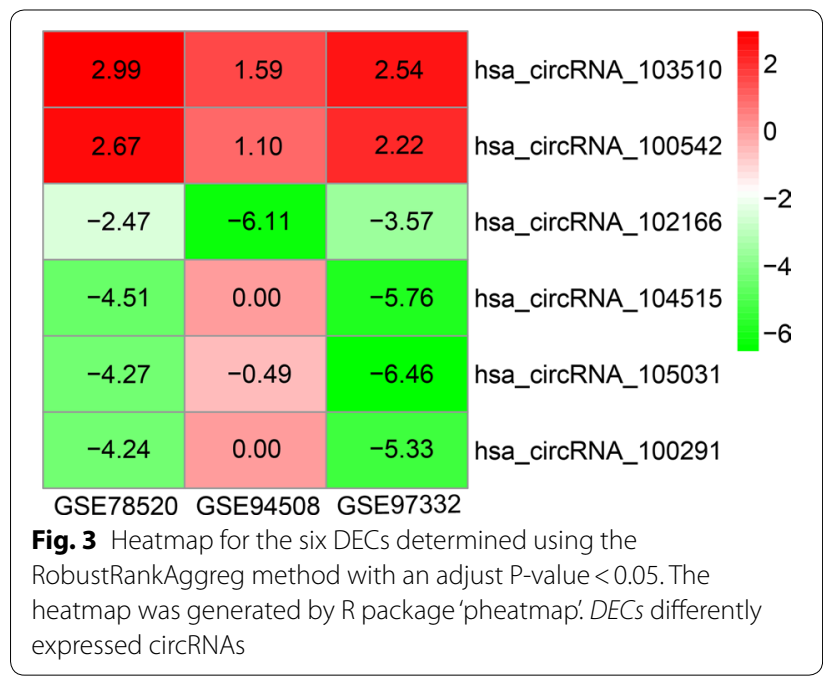

\section{CMap analysis}

Hubgenes consisting of two lists of up- and down-regulated tags were uploaded into the CMap web tool, matching against over 7000 gene expression profiles following treatment of 1309 bioactive compounds in human cell lines [25]. The match between the signatures of interest and chemicals from CMap was assessed by a connectivity score from -1 to 1 : a positive score denotes a stimulative effect of compound on the query signatures; while a negative score implicates a repressed effect of a compound on the query signatures.

\section{Results}

\section{Identification of six DECs in HCC based on RobustRankAggreg method}

Three microarray datasets (GSE78520, GSE94508 and GSE97332) were included in our study. All of the three gene chips were from the platform of Agilent-069978 Arraystar Human CircRNA microarray V1. The basic information of the three datasets is concluded in Table 2. A total of 259 DECs with 211 up-regulated circRNAs and 48 down-regulated circRNAs were found in gene chip GSE78520 (Fig. 2a); 299 DECs with 46 up-regulated circRNAs and 253 down-regulated circRNAs were determined in gene chip GSE94508 (Fig. 2b); 882 DECs with 429 up-regulated circRNAs and 453 down-regulated circRNAs were identified in gene chip GSE97332 (Fig. 2c). Subsequently, we integrated the DECs of the three datasets and ranked them with a robust method. A total of six circRNAs, including two up-regulated circRNAs (hsa_circRNA_103510 and hsa_circRNA_100542) and four down-regulated circRNAs (hsa_circRNA_102166, hsa_circRNA_104515, hsa_circRNA_105031 and hsa_ circRNA_100291), were found to be in the top rankings with an adjust P-value $<0.05$ (Fig. 3). The expression of 
a

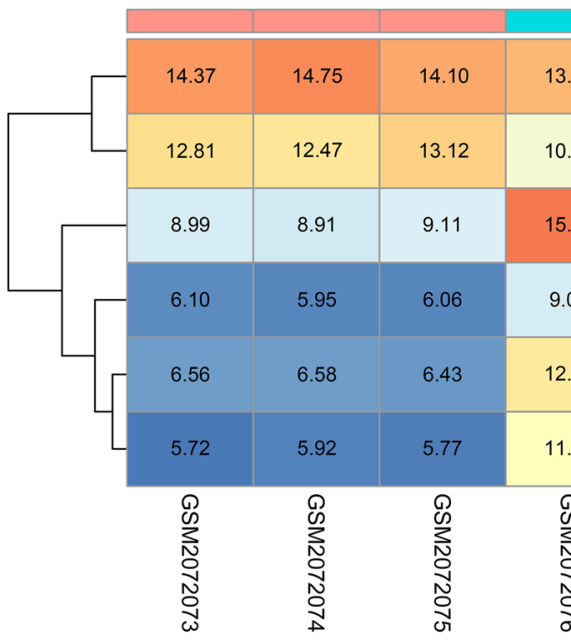

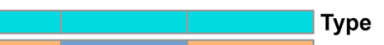

b

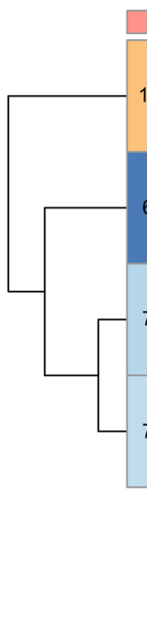

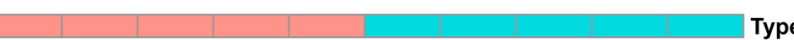

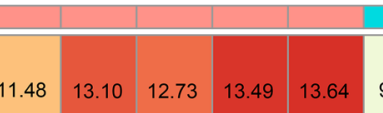

\begin{tabular}{l|l|l|l}
9.16 & 11.51 & 11.85 & 12.70
\end{tabular}

10.07

Type

circRNA_103510

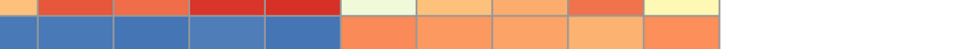

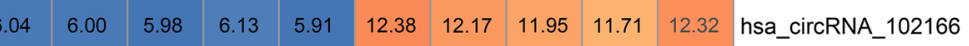

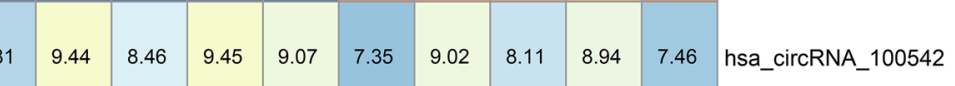

\begin{tabular}{|l|l|l|l|l|l|l|l|l|l|l}
\hline 98 & 7.67 & 7.53 & 7.36 & 7.55 & 8.77 & 8.46 & 8.49 & 8.48 & 8.72 & hsa_circRNA_105031
\end{tabular}

c

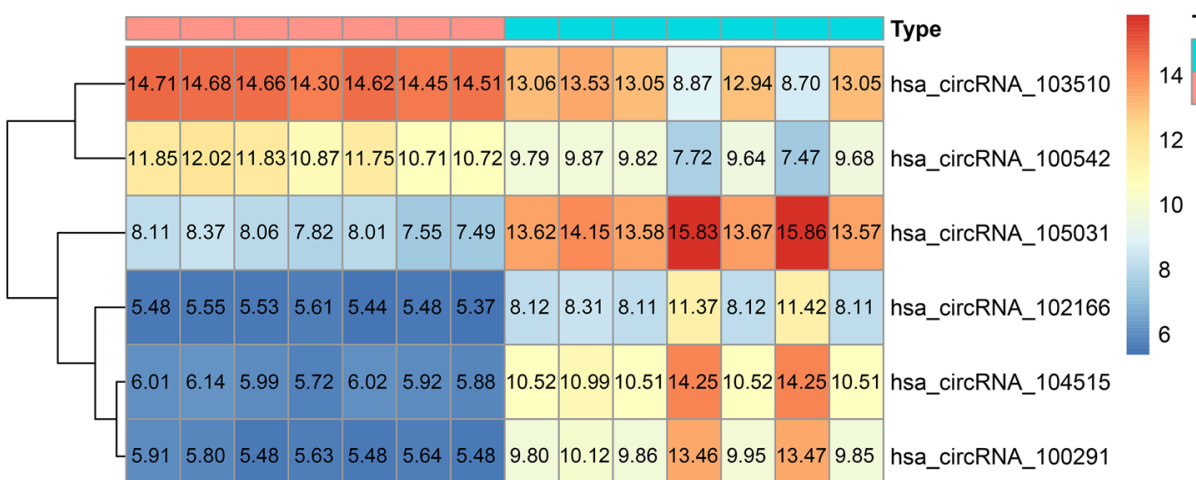

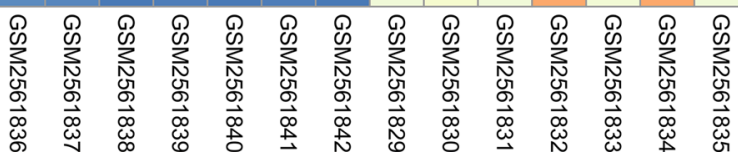

Fig. 4 Heatmap for the six DECs in individual microarray datasets: a GSE78520, b GSE94508, c GSE97332. The heatmap was generated by R package 'pheatmap.' DECs differently expressed circRNAs 
Table 3 Essential characteristics of the six differently expressed circRNAs

\begin{tabular}{|c|c|c|c|c|c|c|c|}
\hline CircRNA & Alias & CircRNA type & Position & Strand & Best transcript & Gene symbol & Regulation \\
\hline $\begin{array}{l}\text { hsa_cir- } \\
\text { CRNA_103510 }\end{array}$ & hsa_circ_0067934 & Exonic & $\begin{array}{c}\text { chr3:170013698- } \\
170015181\end{array}$ & + & uc003fgs.2 & PRKCl & Up \\
\hline $\begin{array}{l}\text { hsa_cir- } \\
\text { CRNA_100542 }\end{array}$ & hsa_circ_0017639 & Exonic & $\begin{array}{l}\text { chr10:7290509- } \\
7327916\end{array}$ & - & uc010qay.2 & SFMBT2 & Up \\
\hline $\begin{array}{l}\text { hsa_cir- } \\
\text { CRNA_102166 }\end{array}$ & hsa_circ_0004913 & Exonic & $\begin{array}{l}\text { chr17:62248459- } \\
62265775\end{array}$ & - & uc002jec.3 & TEX2 & Down \\
\hline $\begin{array}{l}\text { hsa_cir- } \\
\text { CRNA_104515 }\end{array}$ & hsa_circ_0002980 & Exonic & $\begin{array}{c}\text { chr7:141336759- } \\
141349133\end{array}$ & + & uc003vwi.2 & AGK & Down \\
\hline $\begin{array}{l}\text { hsa_cir- } \\
\text { CRNA_105031 }\end{array}$ & hsa_circ_0091570 & Exonic & $\begin{array}{c}\text { chrX:131516205- } \\
131526362\end{array}$ & - & uc004ewt.3 & MBNL3 & Down \\
\hline $\begin{array}{l}\text { hsa_cir- } \\
\text { CRNA_100291 }\end{array}$ & hsa_circ_0000098 & Exonic & $\begin{array}{c}\text { Chr1:101372407- } \\
101387397\end{array}$ & + & uc001dtn.2 & SLC30A7 & Down \\
\hline
\end{tabular}

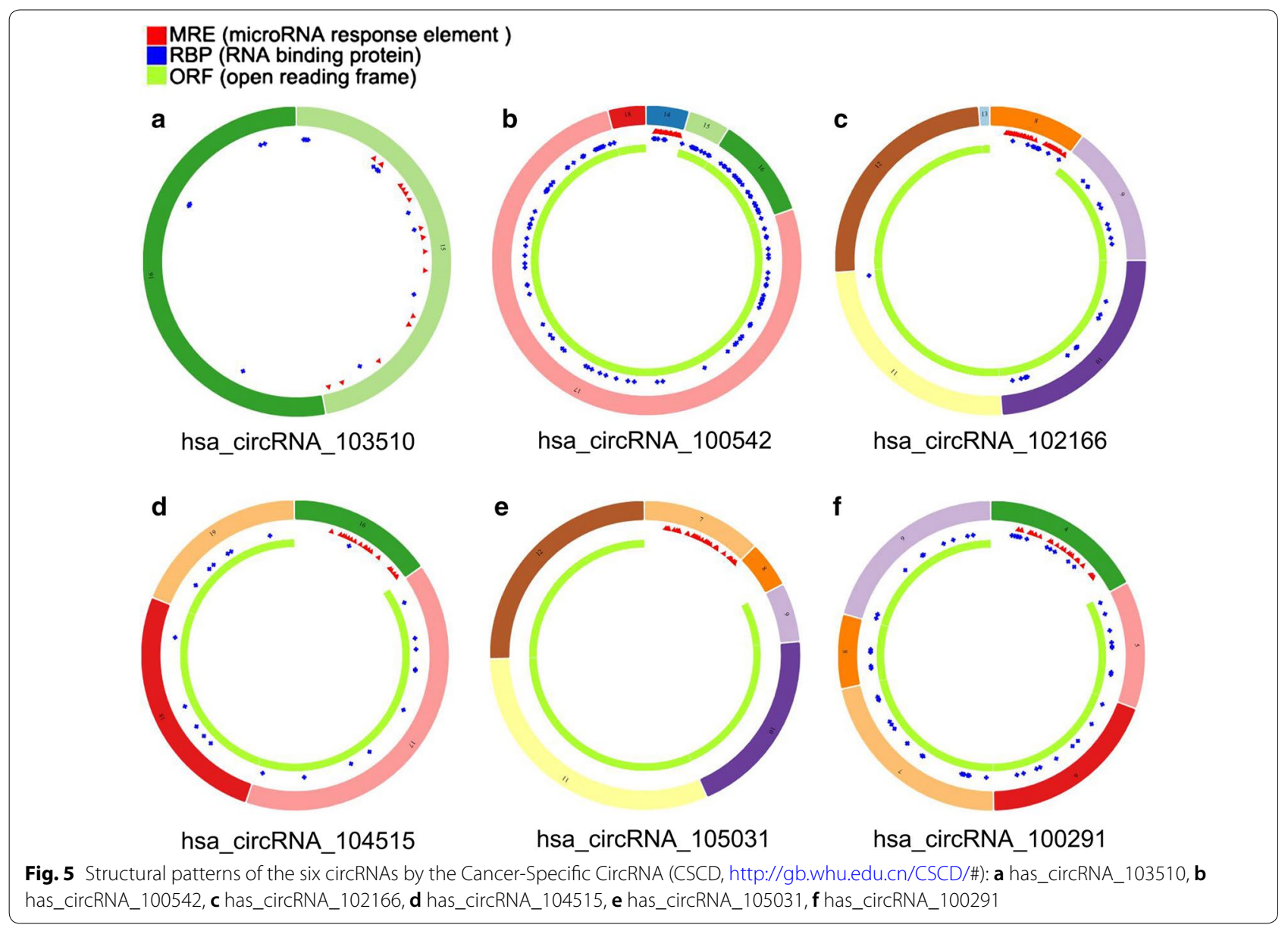



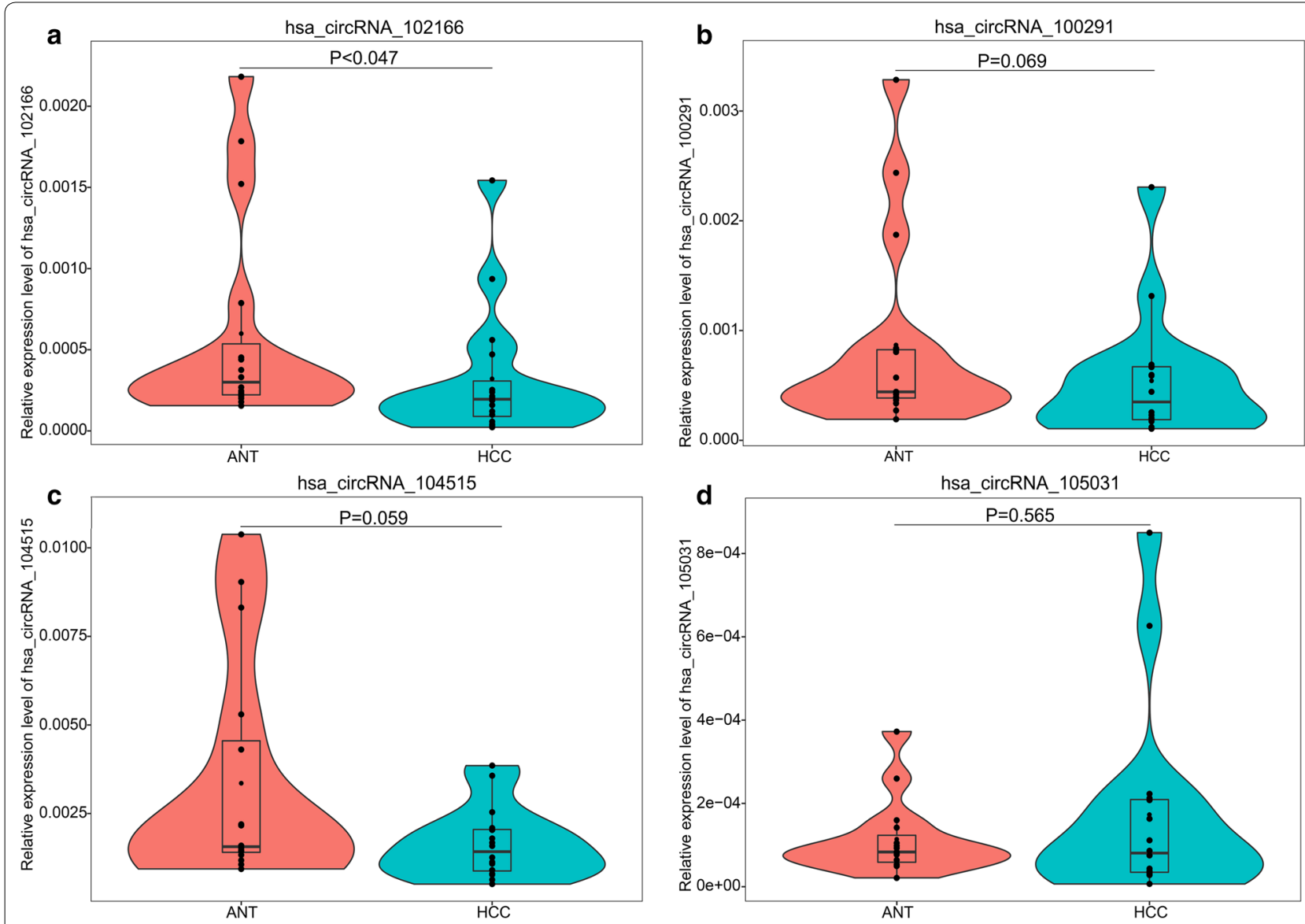

Fig. 6 Violin plots for the expression of hsa_circRNA_102166, hsa_circRNA_104515, hsa_circRNA_105031 and hsa_circRNA_100291 in HCC by RT-qPCR: a hsa_circRNA_102166, b hsa_circRNA_100291, c hsa_circRNA_104515, d hsa_circRNA_105031. HCC hepatocellular carcinoma, ANT adjacent non-tumorous, $R T$ - $q P C R$ reverse transcription-quantitative polymerase chain reaction

the six circRNAs in each dataset is shown in Fig. 4. The essential characteristics of the six circRNAs are displayed in Table 3. The basic structural patterns of the six circRNAs are exhibited in Fig. 5.

\section{Corroboration of the six circRNAs with RT-qPCR}

RT-qPCR was conducted in 16 pairs HCC samples and adjacent non-cancerous tissues to corroborate the expression of the six circRNAs. Among the six circRNAs, hsa_circRNA_103510 and hsa_circRNA_100542 cannot be detected in these in-house tissues due to their relative low expression levels. The low expression of hsa_circRNA_102166 in HCC tissues was corroborated by RT-qPCR ( $\mathrm{P}=0.047$, Fig. 6a). Hsa_circRNA_100291 and hsa_circRNA_104515 showed a down-regulated tendency in $\mathrm{HCC}$, though the P-values were greater than 0.05 (hsa_circRNA_100291: $\mathrm{P}=0.069$, Fig. 6b; hsa_circRNA_104515: $\mathrm{P}=0.059$, Fig. 6c). For hsa_circRNA_105031, its expression in HCC tissues was similar to that in non-cancerous tissues $(\mathrm{P}=0.588$, Fig. $6 \mathrm{~d})$. The head-to-tail splicing in the RT-qPCR product of hsa_circRNA_102166, hsa_circRNA_100291, hsa_circRNA_104515 and hsa_circRNA_105031 was confirmed by Sanger sequencing (Fig. 7).

\section{Identification of five circRNA-miRNA interactions}

Hsa_circRNA_102166, hsa_circRNA_100291 and hsa_circRNA_104515 were selected for further analysis. Increasing evidence has demonstrated that some circRNAs play critical roles in tumors by functioning as "decoys" to sponge miRNAs. To depict whether the three circRNAs perform the similar role in HCC, we 


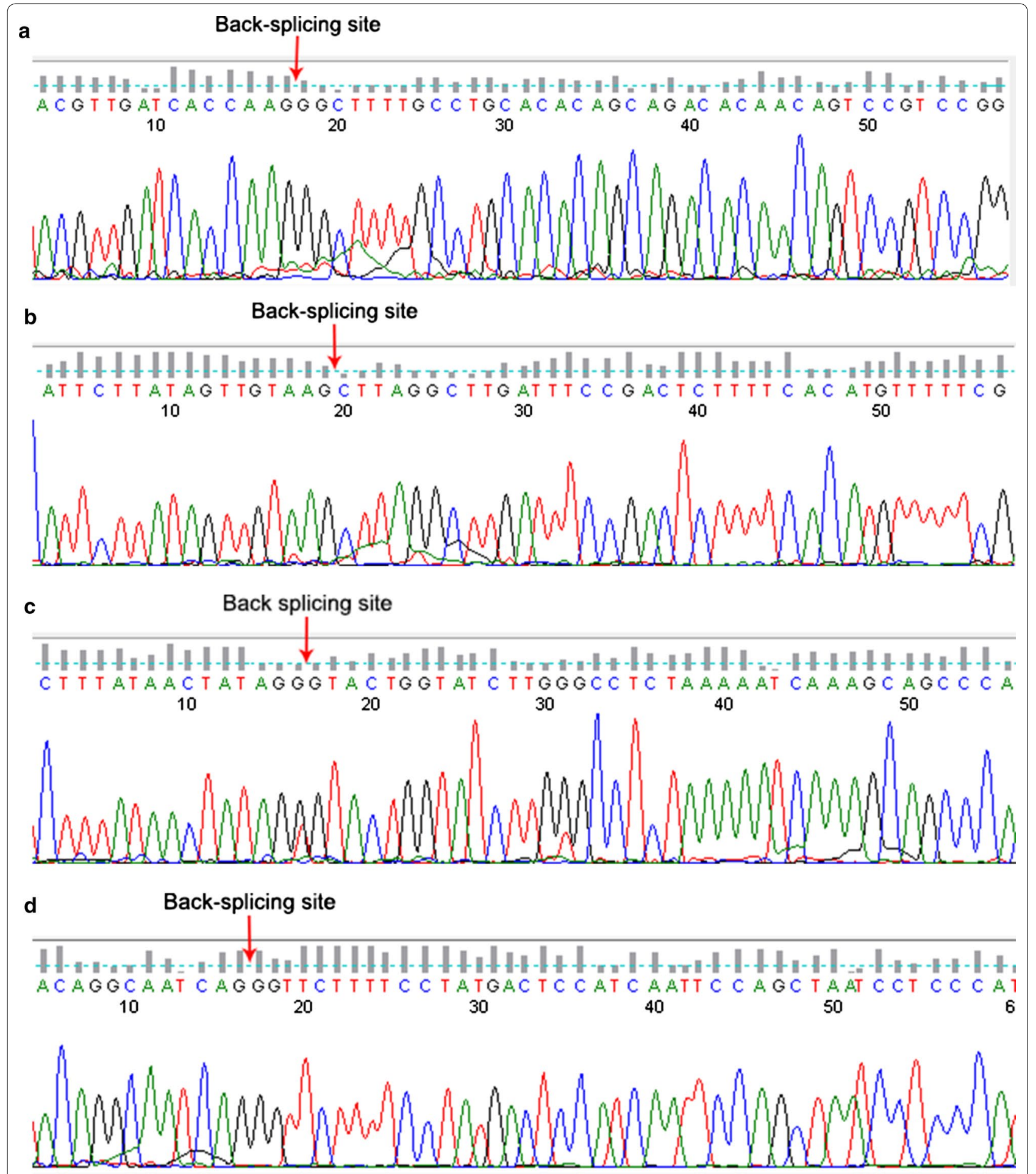

Fig. 7 Head-to-tail splicing in the RT-qPCR product of hsa_circRNA_102166, hsa_circRNA_100291, hsa_circRNA_104515 and hsa_circRNA_105031 by Sanger sequencing: a hsa_circRNA_102166, b hsa_circRNA_100291, chsa_circRNA_104515, d hsa_circRNA_105031 
a
hsa_circRNA_104515 (5' ... 3') ACUGGUAUCUUGGGCCUCUAAAA
hsa-miR-1303 (3' ... 5')
UCUCGUUCUGGGGCAGAGAUUU

b

hsa_circRNA_104515 (5' ... 3') CCAUCAGCAAAGGAGACUUUAUA

hsa-miR-142-5p (3' ... 5')

UCAUCACGAAAGAUGAAAUAC

C
hsa_circRNA_104515 (5' ... 3') UACUGGUAUCUUGGGCCUCUAAA
hsa-miR-877-5p (3' ... 5')
GGGACGCGGUAGAGGAGAUG

e

hsa_circRNA_100291 (5' ... 3') GGACACAGUCAUUCCCUCUUUAA

hsa-miR-1276 (3' ... 5')

\section{ACAGAGGUGUCCCGAGAAAU}

Fig. 8 CircRNA-miRNA interactions identified by both databases of CSCD (http://gb.whu.edu.cn/CSCD/\#) and Circlnteractome (https://circi nteractome.nia.nih.gov/index.html): a hsa_circRNA_104515/hsa-miR-1303, b hsa_circRNA_104515/hsa-miR-142-5p, c hsa_circRNA_104515/ hsa-miR-877-5p, d hsa_circRNA_100291/hsa-miR-583, e hsa_circRNA_100291/hsa-miR-1276. CSCD: Cancer-Specific CircRNA; Circlnteractome: Circular RNA Interactome

collected their potential target miRNAs from two online databases, CSCD and CircInteractome. A total of five circRNA-miRNA interactions including two circRNAs (hsa_circRNA_104515 and hsa_circRNA_100291) and five miRNAs (hsa-miR-1303, hsa-miR-142-5p, hsa-miR877-5p, hsa-miR-583 and hsa-miR-1276) were identified (Fig. 8). DIANA-miRPath [26] was exploited to explore the signaling pathways in which the five miRNAs may be involved. As shown in Fig. 9, all of the five miRNAs were closely linked with some cancer-related pathways.
Expression of the five miRNAs based on the data from GEO and TCGA

In all, 11 microarray and RNA-seq datasets were included. Among them, 7 datasets with 339 HCC samples and 113 normal controls were for miR-1303; 11 datasets with $881 \mathrm{HCC}$ samples and 351 normal controls were for miR-142-5p; 7 datasets with 671 HCC samples and 149 normal controls were for miR-877-5p; 5 datasets with $307 \mathrm{HCC}$ samples and 97 normal controls were for miR-583; and 5 datasets with 534 HCC samples and 110 normal controls were for miR-1276. The essential properties of the 11 records are concluded in Table 4. According 


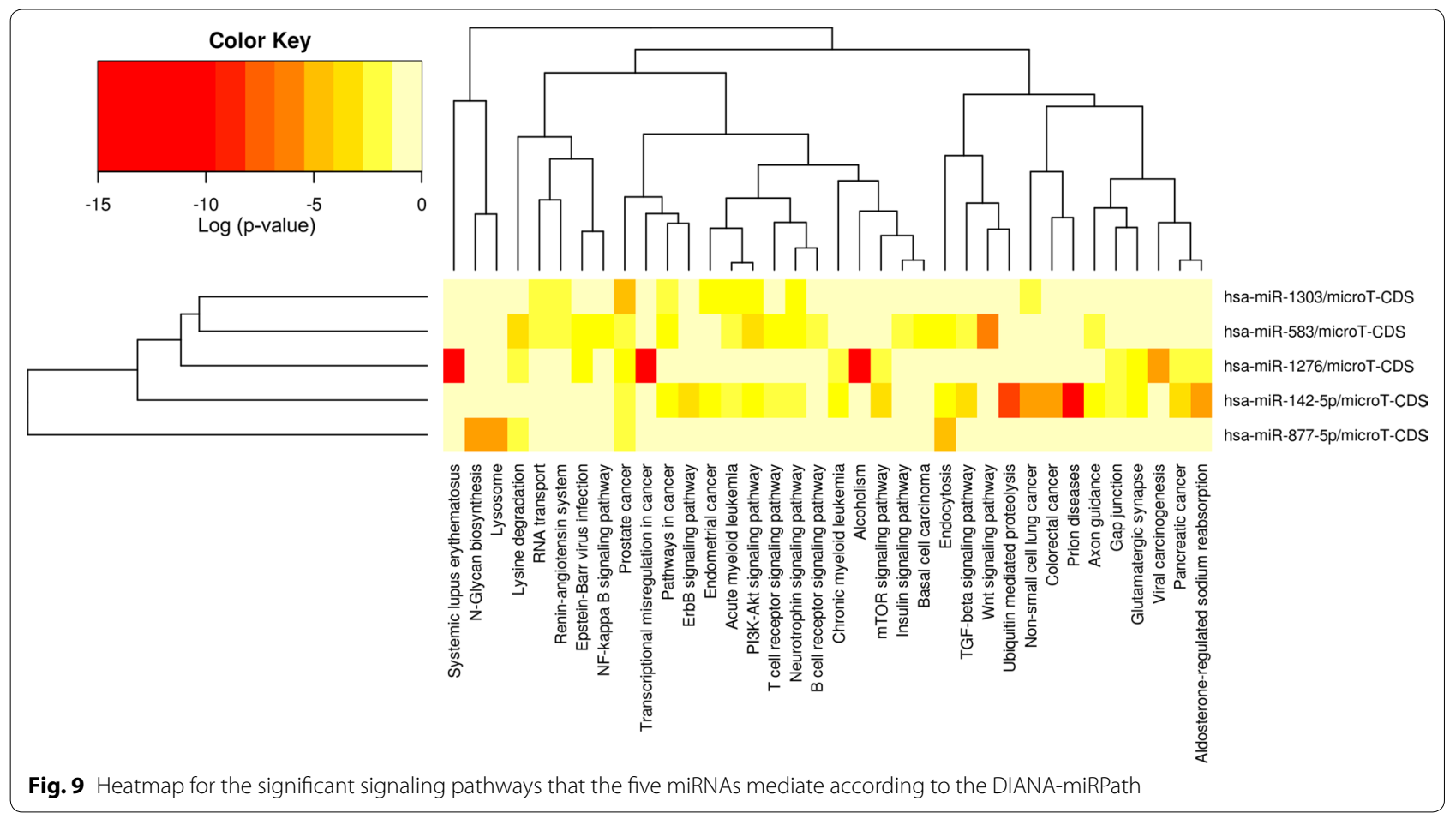

to the pooled results, miR-142-5p was down-regulated $(\mathrm{SMD}=-0.53,95 \% \mathrm{CI}-0.88$ to $-0.19, \mathrm{P}=0.003$; Fig. 10a) and miR-877 was up-regulated $(\mathrm{SMD}=0.80$, 95\% CI $0.25-1.34, \mathrm{P}=0.004$; Fig. 10b) in HCC. MiR1303 and miR-1276 showed high expression tendencies in HCC tissues compared to in normal liver tissues, while the P-values were greater than 0.05 (miR-1303: $\mathrm{SMD}=0.49,95 \% \mathrm{CI}-0.19$ to $1.16, \mathrm{P}=0.157$; Fig. 10c; miR-1276: $S M D=0.21,95 \% \mathrm{CI}-0.30$ to $0.72, \mathrm{P}=0.422$; Fig. 10d). For miR-583, its expression in HCC tissues was similar to that in normal liver tissues $(\mathrm{SMD}=0.03,95 \%$ $\mathrm{CI}-0.25$ to $0.31, \mathrm{P}=0.851$, Fig. $10 \mathrm{e})$.

\section{Construction of circRNA-miRNA-mRNA network}

Total 1424 target genes of the aforementioned five miRNAs were obtained from the miRWalk. Additionally, 3278 DEGs in HCC were gained from the TCGA (Fig. 11a). By intersecting the predicted target genes and DEGs, we identified 172 target genes that exert momentous roles in HCC (Fig. 11b).

We integrated the circRNA-miRNA interactions and miRNA-mRNA interactions to construct a circRNAmiRNA-mRNA network (Fig. 12), which provided a preliminary insight into the links between the DECs (hsa_circRNA_104515 and hsa_circRNA_100291), the five miRNAs (hsa-miR-1303, hsa-miR-142-5p, hsa-miR877-5p, hsa-miR-583 and hsa-miR-1276) and the 172 mRNAs.

\section{Identification of seven hubgenes with MCODE algorithm from PPI network}

Removing unconnected nodes, we established a PPI network consisting of 91 nodes and 131 edges to view the interactions among the 172 mRNAs (Fig. 13a). Considering the importance of hubgene in a network, we employed a MCODE approach to screen hubgenes from the PPI network. With the $\mathrm{k}$-core $=2$, one subnetwork with 7 nodes and 18 edges was identified (Fig. 13b), which unveiled the critical roles of the seven genes (JUN, MYCN, AR, ESR1, FOXO1, IGF1 and CD34) in HCC. The expression levels of the seven genes in HCC are exhibited in Fig. 14. A circRNA-miRNA-hubgene network was then built to delineate the links among the DECs, miRNAs and hubgenes (Fig. 15). Eight circRNAmiRNA-mRNA regulatory modules, including hsa_circRNA_100291/hsa-miR-1276/FOXO1 regulatory axis, hsa_circRNA_100291/hsa-miR-583/ESR1 regulatory axis, hsa_circRNA_100291/hsa-miR-583/JUN regulatory axis, hsa_circRNA_100291/hsa-miR-583/AR regulatory axis, hsa_circRNA_104515/hsa-miR-877-5p/AR regulatory axis, hsa_circRNA_104515/hsa-miR-142-5p/MYCN regulatory axis, hsa_circRNA_104515/has-miR-142-5p/ IGF1 regulatory axis and has_circRNA_104515/has-miR142-5p/CD34 axis, were found from the network. 
Table 4 Essential properties of the 11 records for the five miRNAs by data from GEO

\begin{tabular}{|c|c|c|c|c|c|c|c|c|c|}
\hline \multirow[t]{2}{*}{ miRNA } & \multirow[t]{2}{*}{ First author } & \multirow[t]{2}{*}{ Year } & \multirow[t]{2}{*}{ Region } & \multirow[t]{2}{*}{ Data source } & \multirow[t]{2}{*}{ Platform } & \multicolumn{2}{|c|}{ Number of case } & \multicolumn{2}{|c|}{ Expression (mean $\pm S D$ ) } \\
\hline & & & & & & Cancer & Normal & Cancer & Normal \\
\hline \multirow[t]{7}{*}{ hsa-miR-1303 } & Hou J & 2010 & China & GSE21279 & GPL9052 & 4 & 7 & $4.15 \pm 1.206$ & $2.49 \pm 0.554$ \\
\hline & Sato F & 2011 & Japan & GSE21362 & GPL10312 & 59 & 61 & $1.59 \pm 1.014$ & $1.39 \pm 1.042$ \\
\hline & Kim J & 2012 & South Korea & GSE39678 & GPL15852 & 16 & 8 & $11.00 \pm 0.702$ & $11.49 \pm 0.364$ \\
\hline & $\operatorname{Diaz} \mathrm{G}$ & 2013 & USA & GSE40744 & GPL14613 & 9 & 19 & $3.72 \pm 0.774$ & $2.78 \pm 0.492$ \\
\hline & Villanueva A & 2016 & Spain & GSE74618 & GPL14613 & 230 & 10 & $1.88 \pm 0.658$ & $1.51 \pm 0.263$ \\
\hline & TCGA & 2017 & USA & TCGA & none & 21 & 5 & $0.35 \pm 0.225$ & $0.17 \pm 0.100$ \\
\hline & Xie Z & 2017 & China & GSE98269 & GPL20712 & 3 & 3 & $5.07 \pm 0.071$ & $5.10 \pm 0.115$ \\
\hline \multirow[t]{11}{*}{ hsa-miR-142-5p } & LiW & 2008 & China & GSE10694 & GPL6542 & 78 & 88 & $11.01 \pm 0.605$ & $11.19 \pm 0.710$ \\
\hline & SuH & 2008 & China & GSE12717 & GPL7274 & 9 & 6 & $9.11 \pm 1.406$ & $9.09 \pm 0.707$ \\
\hline & Burchard J & 2010 & USA & GSE22058 & GPL10457 & 96 & 96 & $0.95 \pm 0.241$ & $1.07 \pm 0.135$ \\
\hline & Hou J & 2010 & China & GSE21279 & GPL9052 & 4 & 7 & $7.04 \pm 1.190$ & $7.77 \pm 1.112$ \\
\hline & Sato F & 2011 & Japan & GSE21362 & GPL10312 & 59 & 61 & $7.06 \pm 1.022$ & $7.93 \pm 0.577$ \\
\hline & Kim J & 2012 & South Korea & GSE39678 & GPL15852 & 16 & 8 & $11.10 \pm 0.959$ & $11.88 \pm 0.182$ \\
\hline & Morita K & 2013 & Japan & GSE41874 & GPL7722 & 6 & 4 & $0.72 \pm 0.412$ & $1.34 \pm 0.225$ \\
\hline & Diaz G & 2013 & USA & GSE40744 & GPL14613 & 9 & 19 & $1.96 \pm 0.506$ & $1.74 \pm 0.224$ \\
\hline & Villanueva A & 2016 & Spain & GSE74618 & GPL14613 & 230 & 10 & $1.37 \pm 0.206$ & $1.36 \pm 0.212$ \\
\hline & Xie Z & 2017 & China & GSE98269 & GPL20712 & 3 & 3 & $6.76 \pm 0.733$ & $6.88 \pm 0.169$ \\
\hline & TCGA & 2017 & USA & TCGA & None & 371 & 49 & $5.45 \pm 1.264$ & $6.86 \pm 0.796$ \\
\hline \multirow[t]{7}{*}{ hsa-miR-877-5p } & Hou J & 2010 & China & GSE21279 & GPL9052 & 4 & 6 & $3.64 \pm 1.753$ & $2.19 \pm 0.713$ \\
\hline & Sato F & 2011 & Japan & GSE21362 & GPL10312 & 59 & 61 & $2.17 \pm 0.921$ & $2.05 \pm 0.961$ \\
\hline & Morita K & 2013 & Japan & GSE41874 & GPL7722 & 6 & 4 & $1.08 \pm 0.313$ & $1.06 \pm 0.452$ \\
\hline & Diaz G & 2013 & USA & GSE40744 & GPL14613 & 9 & 19 & $5.36 \pm 0.619$ & $3.88 \pm 0.797$ \\
\hline & Villanueva A & 2016 & Spain & GSE74618 & GPL14613 & 230 & 10 & $3.64 \pm 0.741$ & $3.42 \pm 0.636$ \\
\hline & Xie Z & 2017 & China & GSE98269 & GPL20712 & 3 & 3 & $5.31 \pm 0.073$ & $5.12 \pm 0.066$ \\
\hline & TCGA & 2017 & USA & TCGA & None & 360 & 46 & $1.47 \pm 0.807$ & $0.73 \pm 0.328$ \\
\hline \multirow[t]{5}{*}{ hsa-miR-583 } & Sato F & 2011 & Japan & GSE21362 & GPL10312 & 59 & 61 & $1.47 \pm 0.722$ & $1.46 \pm 0.837$ \\
\hline & Morita K & 2013 & Japan & GSE41874 & GPL7722 & 6 & 4 & $0.91 \pm 0.249$ & $0.76 \pm 0.293$ \\
\hline & $\operatorname{Diaz} \mathrm{G}$ & 2013 & USA & GSE40744 & GPL14613 & 9 & 19 & $1.75 \pm 0.247$ & $1.77 \pm 0.344$ \\
\hline & Villanueva A & 2016 & Spain & GSE74618 & GPL14613 & 230 & 10 & $1.43 \pm 0.237$ & $1.45 \pm 0.294$ \\
\hline & Xie Z & 2017 & China & GSE98269 & GPL20712 & 3 & 3 & $5.07 \pm 0.119$ & $5.02 \pm 0.020$ \\
\hline \multirow[t]{5}{*}{ hsa-miR-1276 } & Sato F & 2011 & Japan & GSE21362 & GPL10312 & 59 & 61 & $1.55 \pm 0.810$ & $1.72 \pm 0.737$ \\
\hline & $\operatorname{Diaz} G$ & 2013 & USA & GSE40744 & GPL14613 & 9 & 19 & $1.9 \pm 0.254$ & $1.70 \pm 0.209$ \\
\hline & Villanueva A & 2016 & Spain & GSE74618 & GPL14613 & 230 & 10 & $1.34 \pm 0.164$ & $1.38 \pm 0.191$ \\
\hline & Xie Z & 2017 & China & GSE98269 & GPL20712 & 3 & 3 & $5.06 \pm 0.049$ & $5.06 \pm 0.027$ \\
\hline & TCGA & 2017 & USA & TCGA & None & 233 & 17 & $0.61 \pm 0.380$ & $0.36 \pm 0.233$ \\
\hline
\end{tabular}

TCGA The Cancer Genome Atlas, SD standard deviation

GO annotation, KEGG pathway and Reactome pathway analyses of the seven hubgenes

$\mathrm{GO}$ analysis was carried out to illustrate the functional annotations of the seven hubgenes. The top five highly enriched GO terms of biological process (BP), cellular component (CC) and molecular function (MF) are shown in Fig. 16. The most enriched GO terms in BP was "epithelial cell proliferation" $(\mathrm{P}<0.0001)$, that in $\mathrm{CC}$ was "chromatin" $(\mathrm{P}<0.0001)$, and that in MF was "betacatenin binding" $(\mathrm{P}<0.0001)$. KEGG pathway analysis was conducted to ascertain the signaling cascade that the seven genes participate. With an adjust $\mathrm{P}$-value $<0.05,12$ 


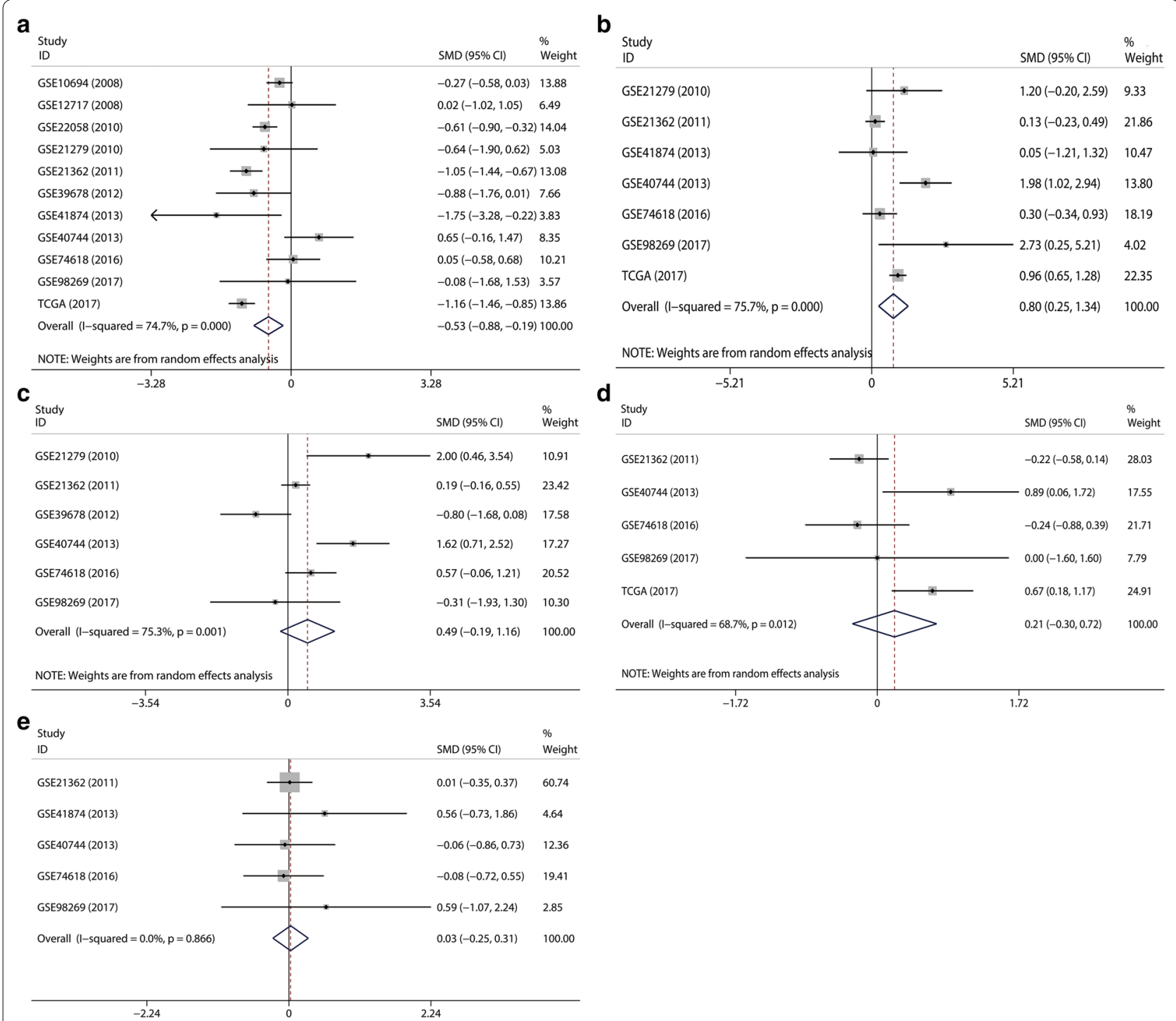

Fig. 10 Forest plots of datasets evaluating the expression of the five miRNAs in hepatocellular carcinoma: a hsa-miR-142-5p, b hsa-miR-877-5p, c hsa-miR-1303, d hsa-miR-1276, e hsa-miR-583

significantly enriched pathways were obtained (Fig. 17a). Among the 12 pathways, "AMPK signaling pathway", "FoxO signaling pathway" and "Estrogen signaling pathway" are linked with the progression of HCC [27-29]. Additionally, some other pathways such as "Prostate cancer", "Breast cancer" and "Transcriptional misregulation in cancer" were also tumor-related pathways. Reactome pathway analysis was further performed to delineate the metabolic pathways that the seven hubgenes related to. With a P-value $<0.05$, a total of 29 pathways were identified (data not shown). The top 10 significantly enriched Reactome pathways are displayed in Fig. 17b.

\section{Identification of three bioactive compounds} for the treatment of HCC based on CMap analysis

The seven hubgenes consisting of two up-regulated genes (CD34, MYCN) and five down-regulated genes (AR, JUN, ESR1, FOXO1, IGF1) were loaded into the CMap web tool as up-regulated tags and down-regulated tags, respectively. Following the signature query, three compounds (decitabine, BW-B70C, gefitinib) with the highest negative enrichment score were determined as the potential therapeutic agents for HCC (Table 5). The chemical structures of the three compounds are presented in Fig. 18. 


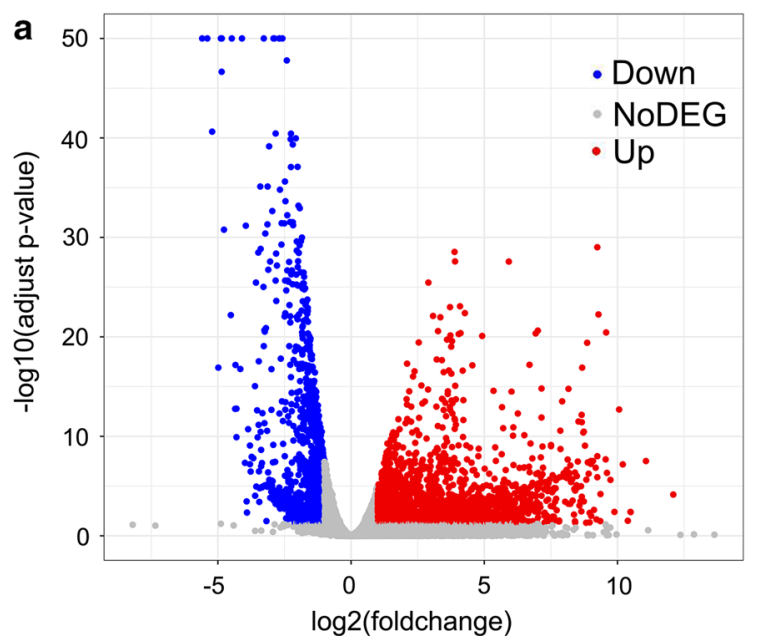

b

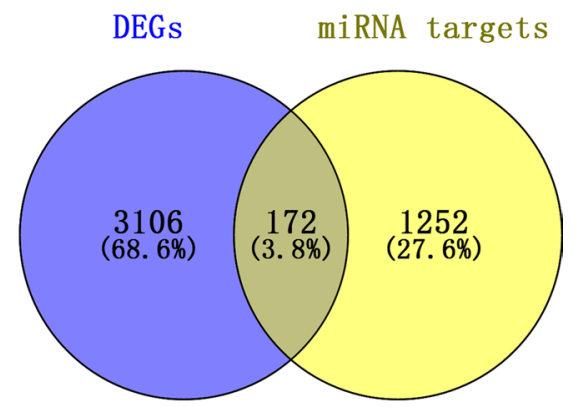

Fig. 11 Identification of 172 genes that exert momentous roles in hepatocellular carcinoma (HCC). a Volcano plot of the differentially expressed genes (DEGs) in HCC based on data from TCGA. The volcano plot was generated by R package 'ggplot2'. b Venn diagram for the intersections between DEGs and miRNA target genes

\section{Discussion}

Due to the lack of $5^{\prime}$ caps and 3'polyadenylated tails, circRNAs are ignored in classical polyadenylated transcriptome studies for a long time. In the past few decades, with the development of high-throughput sequencing, biochemical and computational biology methods, a large number of circRNAs are unmasked the veil in various tissues and cells [30]. Increasing study has unveiled the important roles of circRNAs in a myriad of human diseases, including malignant tumors [31-33]. CircRNAs usually serve as diagnostic and prognostic biomarkers because of their relative tolerance to exonucleases, which benefits from the covalently closed loop structures [34, 35]. In addition, owing to the high cell- and tissue- specificity of circRNAs, their roles in different neoplasms are not complete accord. In HCC, an increasing number of circRNAs, such as ciRS-7 [36], has_circ_0067934 [12], CDR1as [37] and circMTO1 [11], have been reported to exert momentous roles in regulating pathophysiological process and guiding clinical diagnosis and treatment. However, there are still a lot of circRNAs that need to be unearthed.

In this study, we firstly collected three gene chips (GSE78520, GSE94508 and GSE97332) from the GEO database and screened six DECs (hsa_circRNA_103510,hsa_circRNA_100542, hsa circRNA_102166, hsa_circRNA_104515, hsa_circRNA_105031 and hsa_circRNA_100291) with the RobustRankAggreg approach, an algorithm for integration of different gene expression spectra, ranking genes by their fold changes and aggregating these rankings to achieve the final robust and rigorous results. Following the RT-qPCR validation of the six DECs, three circRNAs (hsa_circRNA_102166, hsa_circRNA_100291 and hsa_ circRNA_104515) were selected for further analysis.

As highly conserved endogenous RNAs, many circRNAs harbors abundant miRNA binding sites, indicating that they can sponge corresponding miRNAs and thus function as ceRNAs to regulate gene expression $[8$, 38, 39]. To ascertain whether the aforementioned three circRNAs function as ceNAs in HCC, we predicted their MREs via two online tools, CSCD and CircInteractome. The former web tool predicted MREs within $50 \mathrm{bp}$ upstream and downstream of circRNA junction point [16]. The latter web tool predicted MREs based on the TargetScane algorithm, which forecasts MREs by searching for 7 mer or 8 mer complementarity to the $3^{\prime}$ end and the seed region of each miRNA [17, 40]. We chose miRNA predicted by both algorithms as the putative target miRNA for the three circRNAs. Finally, five circRNA-miRNA interactions consisting of two circRNAs (hsa_circRNA_104515 and hsa_circRNA_100291) and five miRNAs (hsa-miR-1303, hsa-miR-142-5p, hsamiR-877-5p, hsa-miR-583 and hsa-miR-1276) were determined. The expression of the five miRNAs was then verified based on the microarray and RNA-seq data from the GEO and TCGA. The results showed that miR142-5p was down-regulated in HCC, which was consistent with previous studies $[41,42]$. No relevant study reported the expression of miR-877, miR-1303, miR-1276 and miR-583 in HCC. According to our results, miR877 was up-regulated in HCC. MiR-1303 and miR-1276 showed high expression tendencies in HCC, while the differences were not statistical significant. For miR-583, 


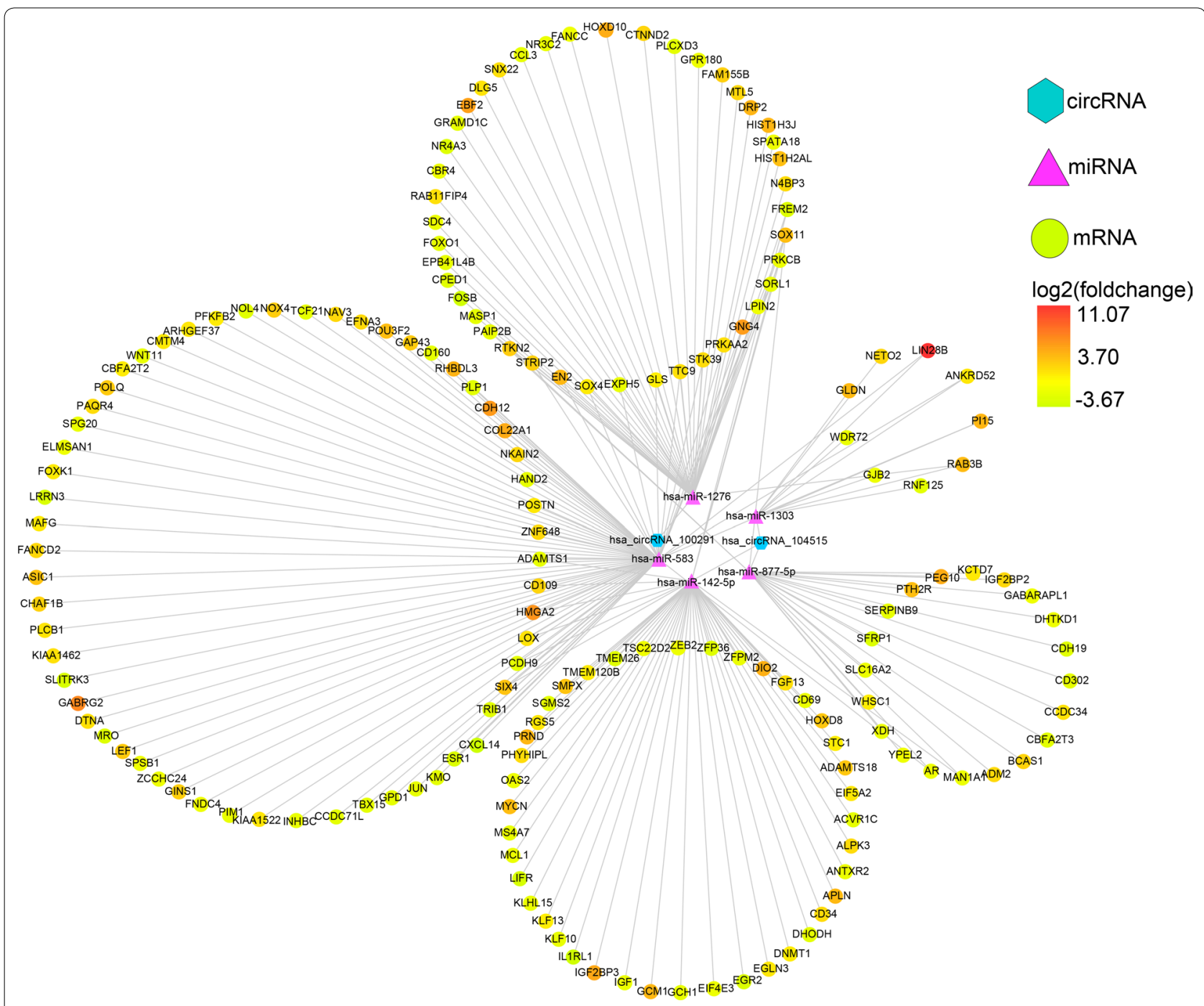

Fig. 12 circRNA-miRNA-mRNA regulatory network. The network consisting of two cricRNAs (hsa_circRNA_104515 and hsa_circRNA_100291), five miRNAs (hsa-miR-1303, hsa-miR-142-5p, hsa-miR-877-5p, hsa-miR-583 and hsa-miR-1276) and 172 genes was generated by Cytoscape 3.6.1

its expression in HCC tissues was similar to that in normal liver tissues. Due to the inter-study heterogeneity that cannot be ignored, the reliable of the pooled results is reduced. Thereby, further rigorous studies are necessary to validate these findings.

Following the collection of the 172 overlapped genes between the target genes of the five miRNAs and the DEGs in HCC, we constructed a circRNA-miRNAmRNA regulatory network. We found that hsa_circRNA_104515 and hsa_circRNA_100291 may act as ceRNAs to capture miR-1276, miR-583, miR-877-5p or miR-142-5p, and subsequently regulate the 172 genes expression. Our results provide an evidence of the ceRNA regulatory mechanism of hsa_circRNA_104515 and hsa circRNA_100291 in HCC. To further elucidate the action mechanism of the ceRNA network, we constructed a PPI network, screening seven hubgenes (JUN, MYCN, AR, ESR1, FOXO1, IGF1 and CD34) from the PPI network. The functional annotations and pathway analyses showed that the seven hubgenes genes were involved in many critical tumor-related biological functions and metabolic pathways, such as "epithelial cell proliferation", "AMPK signaling pathway", "FoxO signaling pathway", "Estrogen signaling pathway", "MAPK6/MAPK4 signaling" and "MAPK family signaling cascades". The important roles of the seven genes in HCC have also been demonstrated previously [43-49]. However, their links with circRNAs have not yet been explored. Here, we identified seven circRNA-miRNA-mRNA axes (hsa_circRNA_100291/ miR-1276/FOXO1, hsa_circRNA_100291/miR-583/ 


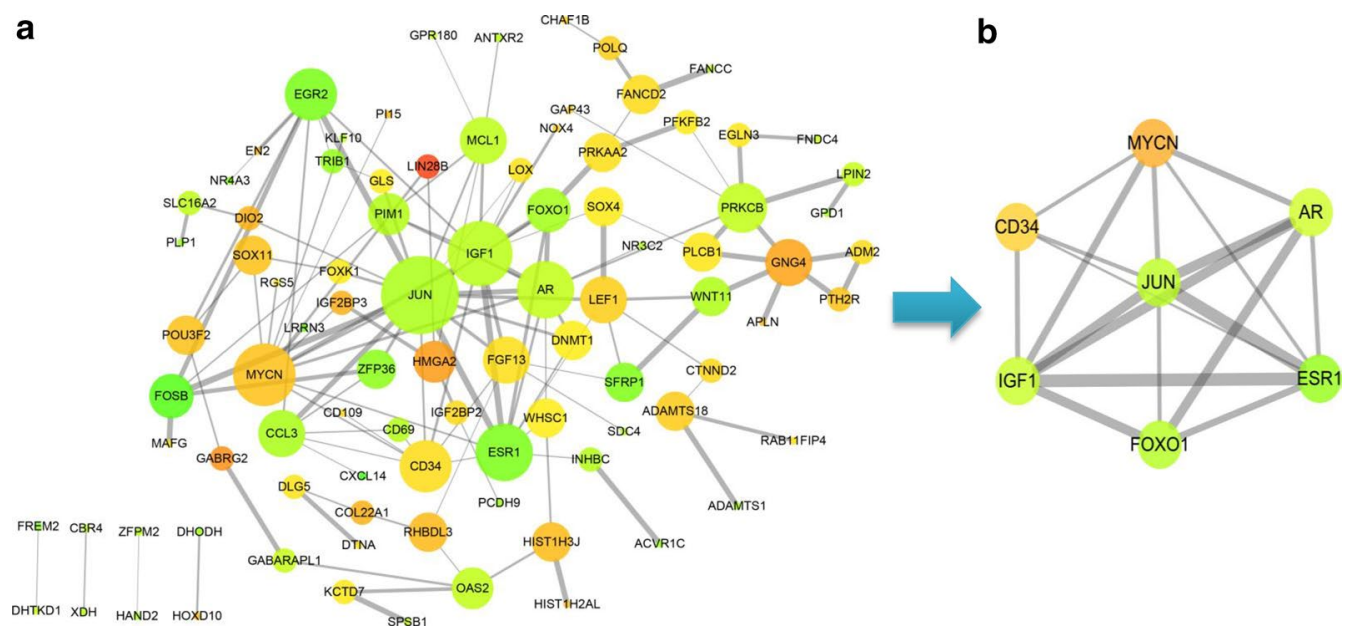

Fig. 13 Identification of hubgenes from the PPI network with the MCODE algorithm. The node color changes gradually from green to red in ascending order according to the log2(foldchange) of genes. The edge size changes gradually from fine to coarse in ascending order according to the combined score between two neighbored genes. a A PPI network of the 172 target genes that exert momentous roles in hepatocellular carcinoma. This network consists of 91 nodes and 131 edges. The node size changes gradually from small to large in ascending order according to the number of neighbored genes per gene. $\mathbf{b}$ A PPI network of the 7 hubgenes that extracted from $\mathbf{a}$. This network consists of 7 nodes and 18 edges. PPI protein-protein interaction, MCODE Molecular Complex Detection
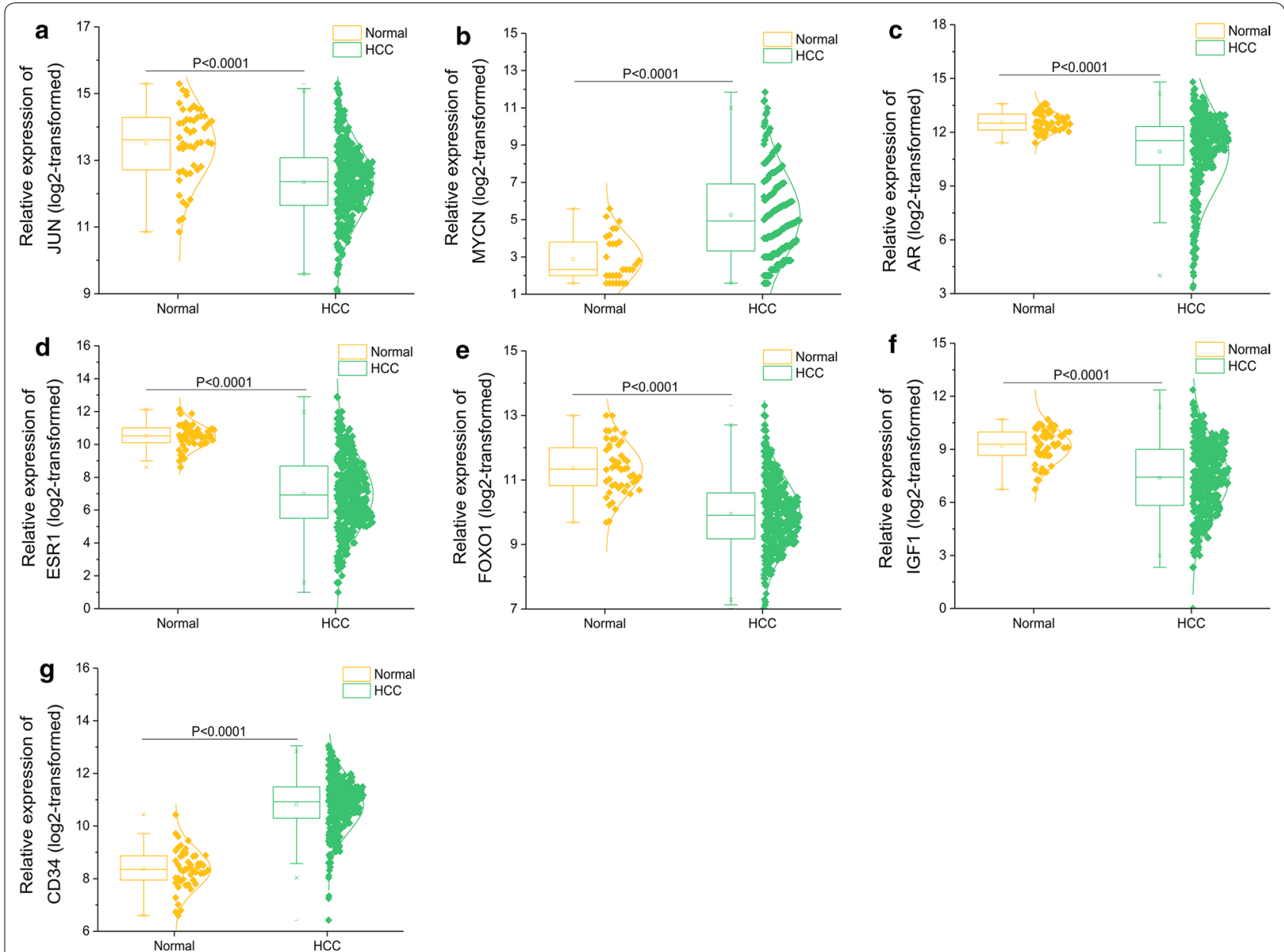

Fig. 14 Box-scatter plots for the seven hubgenes expression in hepatocellular carcinoma (HCC): a JUN, b MYCN, c AR, d ESR1, e FOXO1, f IGF1, g CD34 


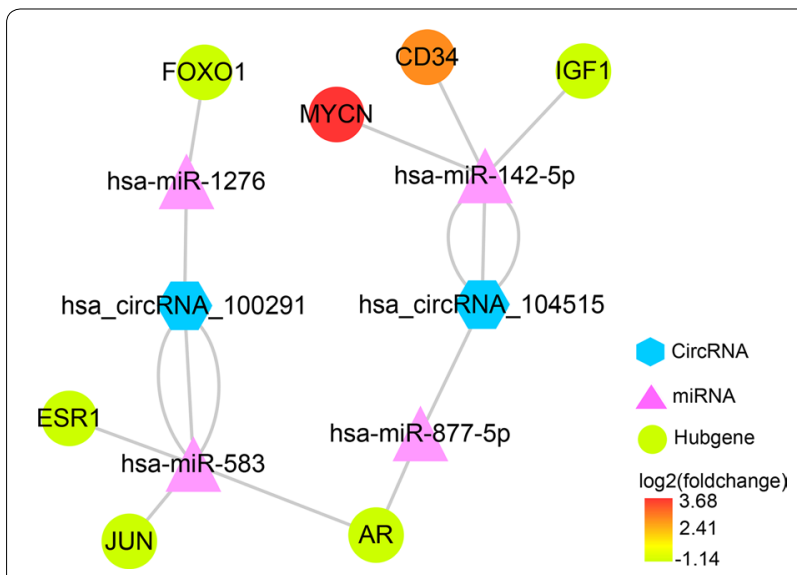

Fig. 15 CircRNA-miRNA-hubgene network. The network consisting of two cricRNAs (hsa_circRNA_104515 and hsa_circRNA_100291), four miRNAs (hsa-miR-142-5p, hsa-miR-877-5p, hsa-miR-583 and hsa-miR-1276) and seven hubgenes (JUN, MYCN, AR, ESR1, FOXO1, IGF1 and CD34) was generated by Cytoscape 3.6.1
ESR1, hsa_circRNA_100291/miR-583/JUN, hsa_circRNA_100291/miR-583/AR, hsa_circRNA_104515/ miR-877-5p/AR, hsa_circRNA_104515/miR-142-5p/ MYC, hsa_circRNA_104515/miR-142-5p/MYCN, hsa_circRNA_104515/miR-142-5p/IGF1 and hsa_circRNA_104515/miR-142-5p/CD34), indicating competitive regulatory relationships of hsa_circRNA_100291 and hsa_circRNA_104515 with the seven genes in HCC. However, given that the results are on the basis of computational biology, further in-depth studies are indispensable to verify the possible roles of the seven axes in HCC.

To date drug control is an important treatment for patients with HCC [50]. Digging effective and sensitive drugs against HCC helps improve patients' outcomes. We therefore implemented the CMap analysis of the seven hubgenes to explore usable drugs for the treatment of HCC. Based on the genome-wide expression profiling of transcripts technology, CMap provides a

a Regulation of fibroblast proliferation-

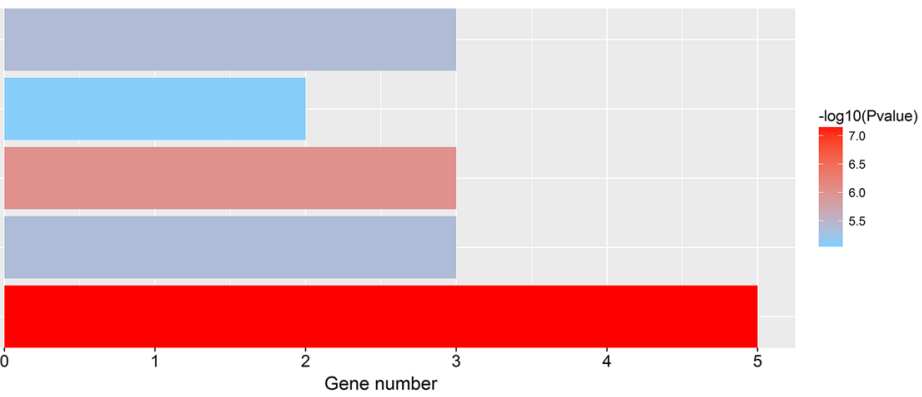

b

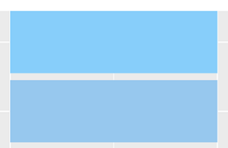

Nuclear chromosome part

Pore complex

Nuclear chromatin-

Chromatin-
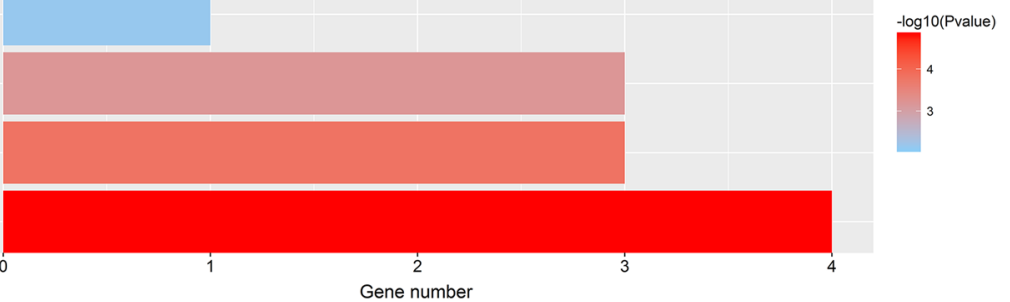

C

Transcriptional activator activity, RNA polymerase II core promoter proximal region sequence-specific binding

Transcription factor activity, RNA polymerase II core promoter proximal region sequence-specific binding

RNA polymerase II transcription factor binding

Chromatin binding
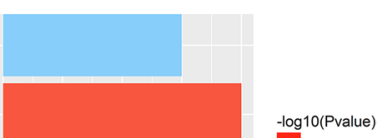

Beta-catenin binding
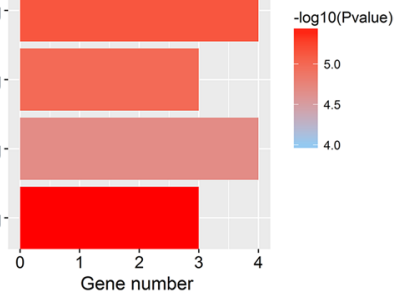

Fig. 16 Top five Gene Ontology (GO) enrichment annotations of the seven hubgenes: a biological process, b cellular component, $\mathbf{c}$ molecular function. GO analysis was conducted by R package 'clusterProfiler' and visualized by R package 'ggplot2' 


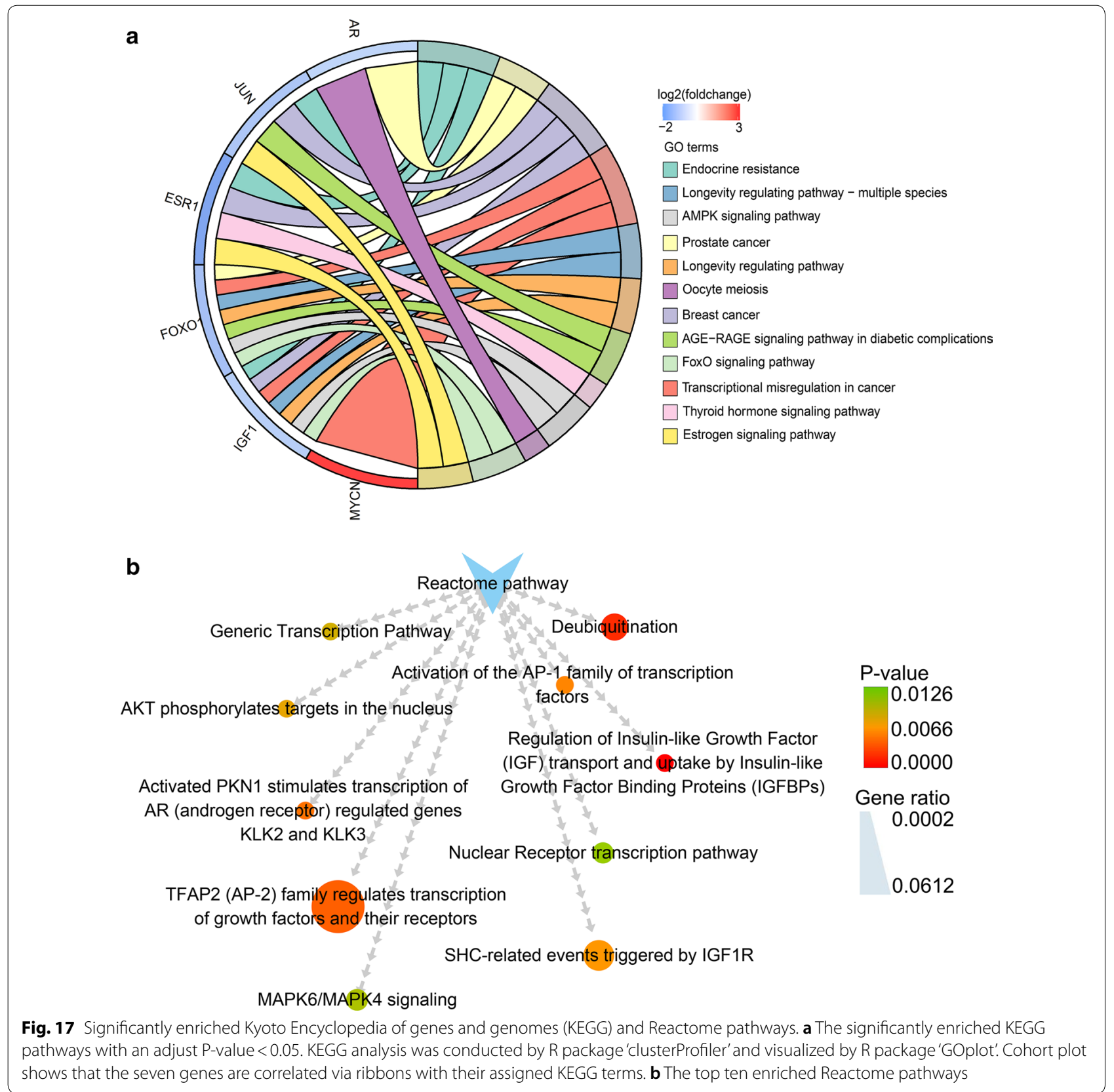

Table 5 Three compounds identified as treatment options for hepatocellular carcinoma by CMap analysis

\begin{tabular}{llllll}
\hline CMap name & $\begin{array}{l}\text { Enrichment } \\
\text { score }\end{array}$ & Dose & Cell & Up score & Down score \\
\hline Decitabine & -0.996 & $100 \mathrm{nM}$ & MCF7 & -0.649 & 0.238 \\
BW-B70C & -0.98 & $32 \mu \mathrm{M}$ & MCF7 & -0.564 & 0.226 \\
Gefitinib & -0.979 & $10 \mu \mathrm{M}$ & HL60 & -0.455 & 0.329 \\
\hline
\end{tabular}

CMap connectivity map comprehensive and accurate data resource for exploration of novel drug or relocation of existing drug [51]. Drugs available in CMap are all licensed for human use by the Food and Drug Administration, thus it is an ideal and reliable approach to obtain therapeutic agents for human diseases [52]. Three chemicals (decitabine, BW$\mathrm{B} 70 \mathrm{C}$ and gefitinib) were identified as the treatment options for HCC. As a cytidine antimetabolite analogue, decitabine represses DNA methylation, arresting cells 

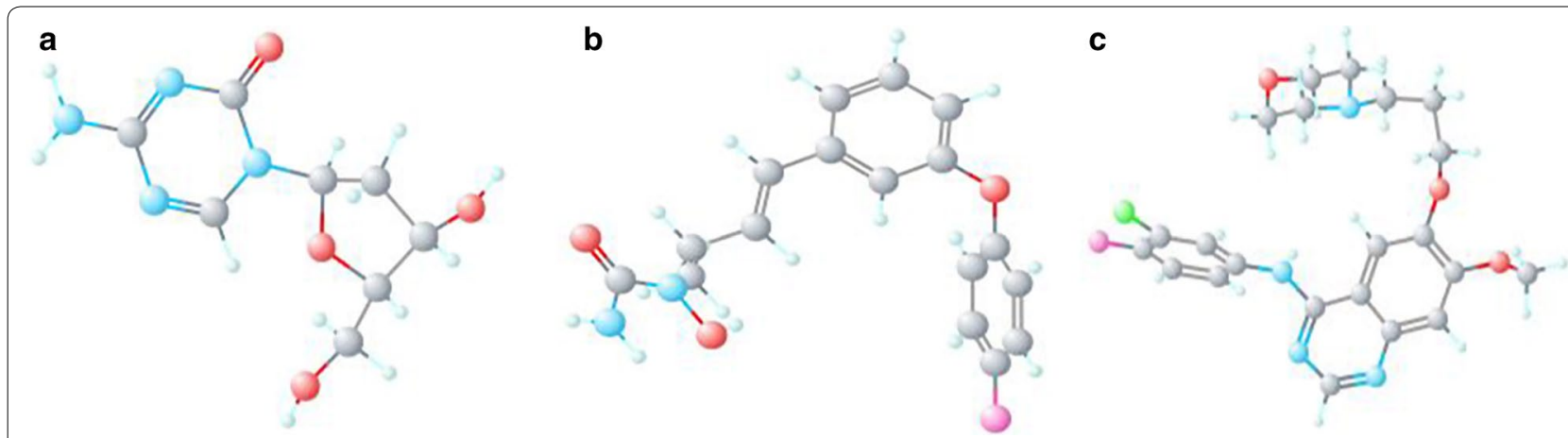

Fig. 18 3D-structure of the three compounds identified by the connectivity map analysis: a decitabine, b BW-B70C, c gefitinib

into G1/S phase and inhibiting tumor cell proliferation. Its antitumor activity in solid tumors including HCC has been elucidated in previous studies [53]. Xing et al. [54] have demonstrated that decitabine could facilitate the expression of miR-122 via inhibition of methylation in HCC cells. More importantly, Skårn et al. [55] have demonstrated that miR-142 is epigenetically restrained by DNA methylation. Treating decitabine in mesenchymal cells promotes mature miR-142-5p/3p expression and thus depresses cell proliferation. We thus hypothesize that decitabine may exert its anti-HCC effect by augmenting miR-142-5p via demethylation and subsequently regulating the downstream target genes of miR-142-5p. Further well-design study is necessary to validate the conclusion. BW-B70C is an inhibitor of arachidonic acid 5-lipoxygenase. Previous study has reported its anti-neoplastic activity in leukemic cells by suppressing the NOTCH1-P13K-AKT-eNOS axis [56]. However, its anti-HCC effect and action mechanism have not been elucidated as of yet. In this study, we found its potential as therapeutic agent for HCC. More studies are needed to verify this finding. Gefitinib is a selective inhibitor of tyrosine kinase receptor used in clinic for the treatment of locally advanced or metastatic non-small cell lung cancer (NSCLC) [57]. Its antineoplastic effect on the other solid tumors including $\mathrm{HCC}$ has also been reported $[58,59]$. However, the responsiveness of different HCC patients to gefitinib varies greatly and most patients even develop gefitinib resistance [60]. Multiple non-coding RNAs including miRNAs and lncRNAs have been reported to contribute to gefitinib resistance and sensitivity in NSCLC [61-63]. We speculate that gefitinib share the similar resistance mechanisms in HCC and lung cancer. Our study provides a theoretical basis for studying gefitinib resistance mechanism and enhancing gefitinib sensitivity in patients with HCC from the perspective of circRNAmiRNA-mRNA network.

\section{Conclusions}

In conclusion, by employing a comprehensive strategy of big data mining, RT-qPCR and computational biology, we constructed a circRNA-miRNA-mRNA network and found that hsa_circRNA_100291 and hsa_circRNA_104515 may function as ceRNAs to exert critical roles in HCC. In addition, three bioactive chemicals (decitabine, BW-B70C and gefitinib) based on the CMap analysis was determined as therapeutic agents for HCC. Our study provides a novel insight into the pathogenesis and therapy for HCC from the circRNA-miRNA-mRNA view.

\section{Abbreviations}

CircRNA: circular RNA; HCC: hepatocellular carcinoma; RT-qPCR: reverse transcription-quantitative polymerase chain reaction; CMap: connectivity map; ceRNA: competing endogenous RNA; MRE: miRNA response element; GEO: Gene Expression Omnibus; DEC: differently expressed circRNA; PPI: proteinprotein interaction; GO: Gene Oncology; KEGG: Kyoto Encyclopedia of Genes and Genomes; CDNA: complementary DNA; CSCD: Cancer-Specific CircRNA; Circlnteractome: Circular RNA Interactome; TCGA: The Cancer Genome Atlas; SMD: standard mean difference; $95 \% \mathrm{Cl}$ : 95\% confidence interval; RNA-seq: RNA-sequencing; MCODE: Molecular Complex Detection; DEG: differently expressed gene; BP: biological process; CC: cellular component; MF: molecular function; FDA: Food and Drug Administration; NSCLC: non-small cell lung cancer.

\section{Authors' contributions}

DDX and YWD collected circRNA-related datasets; screened differently expressed circRNAs; conducted RT-qPCR; collected and analyzed miRNArelated data from TCGA and GEO; and were the major contributors in writing the manuscript. PL and DYW collected miRNA target genes as well as differently expressed genes in HCC; construed PPI network; and conducted GO, KEGG and Reactome analyses. RQH conducted CMap analysis and checked all of the data used in the manuscript. ZBF and GC guided the design of all of the experiments and the writing of the manuscript. All authors read and approved the final manuscript.

\footnotetext{
Author details

${ }^{1}$ Department of Pathology, First Affiliated Hospital of Guangxi Medical University, 6 Shuangyong Road, Nanning 530021, Guangxi Zhuang Autonomous Region, People's Republic of China. ${ }^{2}$ Department of Medical Ultrasonics, First Affiliated Hospital of Guangxi Medical University, 6 Shuangyong Road, Nanning 530021, Guangxi Zhuang Autonomous Region, People's Republic of China. ${ }^{3}$ Department of Medical Oncology, First Affiliated Hospital of Guangxi Medical University, 6 Shuangyong Road, Nanning 530021, Guangxi Zhuang Autonomous Region, People's Republic of China.
} 


\section{Acknowledgements \\ Not applicable.}

\section{Competing interests}

The authors declare that they have no competing interests.

\section{Availability of data and materials}

The datasets used and/or analyzed during the current study are available from the corresponding author on reasonable request.

\section{Consent for publication}

Not applicable.

\section{Ethics approval and consent to participate}

The Ethical Committee of the First Affiliated Hospital of Guangxi Medical University approved the present study.

\section{Funding}

This study was supported by the National Natural Science Foundation of China (NSFC81560489 and NSFC81560386), the Natural Science Foundation of Guangxi, China (2017GXNSFAA198017), the Guangxi Medical University Training Program for Distinguished Young Scholars (2017) and the Medical Excellence Award Funded by the Creative Research Development Grant from the First Affiliated Hospital of Guangxi Medical University (Gang Chen and Yi-wu Dang)

\section{Publisher's Note}

Springer Nature remains neutral with regard to jurisdictional claims in published maps and institutional affiliations.

Received: 28 May 2018 Accepted: 31 July 2018

Published online: 09 August 2018

\section{References}

1. Sanger HL, Klotz G, Riesner D, Gross HJ, Kleinschmidt AK. Viroids are single-stranded covalently closed circular RNA molecules existing as highly base-paired rod-like structures. Proc Natl Acad Sci U S A. 1976;73:3852-6.

2. Wang PL, Bao Y, Yee MC, Barrett SP, Hogan GJ, Olsen MN, et al. Circular RNA is expressed across the eukaryotic tree of life. PLOS ONE. 2014;9:e90859.

3. Salzman J, Chen RE, Olsen MN, Wang PL, Brown PO. Cell-type specific features of circular RNA expression. PLoS Genet. 2013;9:e1003777.

4. Vidal AF, Sandoval GT, Magalhaes L, Santos SE, Ribeiro-dos-Santos A. Circular RNAs as a new field in gene regulation and their implications in translational research. Epigenomics. 2016;8:551-62.

5. Wang Y, Mo Y, Gong Z, Yang X, Yang M, Zhang S, et al. Circular RNAs in human cancer. Mol Cancer. 2017;16:25.

6. Kristensen LS, Hansen TB, Veno MT, Kjems J. Circular RNAs in cancer: opportunities and challenges in the field. Oncogene. 2018;37:555-65.

7. Qi X, Zhang DH, Wu N, Xiao JH, Wang X, Ma W. ceRNA in cancer: possible functions and clinical implications. J Med Genet. 2015;52:710-8.

8. Zhong Y, Du Y, Yang X, Mo Y, Fan C, Xiong F, et al. Circular RNAs function as ceRNAs to regulate and control human cancer progression. Mol Cancer. 2018;17:79

9. Peng L, Yuan XQ, Li GC. The emerging landscape of circular RNA ciRS-7 in cancer (Review). Oncol Rep. 2015;33:2669-74.

10. Geng HH, Li R, Su YM, Xiao J, Pan M, Cai XX, et al. The circular RNA Cdr1as promotes myocardial infarction by mediating the regulation of miR-7a on its target genes expression. PLoS ONE. 2016;11:e0151753.

11. Han D, Li J, Wang H, Su X, Hou J, Gu Y, Qian C, et al. Circular RNA circMTO1 acts as the sponge of microRNA-9 to suppress hepatocellular carcinoma progression. Hepatology. 2017;66:1151-64.

12. Zhu Q, Lu G, Luo Z, Gui F, Wu J, Zhang D, et al. CircRNA circ_0067934 promotes tumor growth and metastasis in hepatocellular carcinoma through regulation of miR-1324/FZD5/Wnt/beta-catenin axis. Biochem Biophys Res Commun. 2018:497:626-32
13. Barrett T, Wilhite SE, Ledoux P, Evangelista C, Kim IF, Tomashevsky M, et al. NCBI GEO: archive for functional genomics data sets-update. Nucleic Acids Res. 2013:41:D991-5.

14. Ritchie ME, Phipson B, Wu D, Hu Y, Law CW, Shi W, et al. Limma powers differential expression analyses for RNA-sequencing and microarray studies. Nucleic Acids Res. 2015;43:e47.

15. Kolde R, Laur S, Adler P, Vilo J. Robust rank aggregation for gene list integration and meta-analysis. Bioinformatics. 2012;28:573-80.

16. Xia S, Feng J, Chen K, Ma Y, Gong J, Cai F, et al. CSCD: a database for cancer-specific circular RNAs. Nucleic Acids Res. 2018;46:D925-9.

17. Dudekula DB, Panda AC, Grammatikakis I, De S, Abdelmohsen K, Gorospe M. Circlnteractome: a web tool for exploring circular RNAs and their interacting proteins and microRNAs. RNA Biol. 2016;13:34-42.

18. Deng M, Bragelmann J, Schultze JL, Perner S. Web-TCGA: an online platform for integrated analysis of molecular cancer data sets. BMC Bioinform. 2016;17:72

19. Dweep H, Gretz N, Sticht C. miRWalk database for miRNA-target interactions. Methods Mol Biol. 2014:1182:289-305.

20. Robinson MD, McCarthy DJ, Smyth GK. edgeR: a Bioconductor package for differential expression analysis of digital gene expression data. Bioinformatics. 2010;26:139-40.

21. Su G, Morris JH, Demchak B, Bader GD. Biological network exploration with Cytoscape 3. Curr Protoc Bioinform. 2014;47:8.13.1-8.1324

22. Bader GD, Hogue CW. An automated method for finding molecular complexes in large protein interaction networks. BMC Bioinform. 2003;4:2.

23. Yu G, Wang LG, Han Y, He QY. clusterProfiler: an R package for comparing biological themes among gene clusters. OMICS. 2012;16:284-7.

24. Wu G, Dawson E, Duong A, Haw R, Stein L. ReactomeFIViz: a Cytoscape app for pathway and network-based data analysis. F1000Res. 2014;3:146.

25. Musa A, Ghoraie LS, Zhang SD, Glazko G, Yli-Harja O, Dehmer M, et al. A review of connectivity map and computational approaches in pharmacogenomics. Brief Bioinform. 2017;18:903.

26. Vlachos IS, Zagganas K, Paraskevopoulou MD, Georgakilas G, Karagkouni D, Vergoulis T, et al. DIANA-miRPath v3.0: deciphering microRNA function with experimental support. Nucleic Acids Res. 2015;43:W460-6.

27. Huang GM, Jiang QH, Cai C, Qu M, Shen W. SCD1 negatively regulates autophagy-induced cell death in human hepatocellular carcinoma through inactivation of the AMPK signaling pathway. Cancer Lett. 2015;358:180-90

28. Hou YQ, Yao Y, Bao YL, Song ZB, Yang C, Gao XL, et al. Juglanthraquinone $C$ induces intracellular ROS increase and apoptosis by activating the Akt/Foxo signal pathway in HCC cells. Oxid Med Cell Longev. 2016;2016:4941623

29. You H, Meng K, Wang ZY. The ER-alpha36/EGFR signaling loop promotes growth of hepatocellular carcinoma cells. Steroids. 2018;134:78-87.

30. Chen LL, Yang L. Regulation of circRNA biogenesis. RNA Biol. 2015:12:381-8

31. Yang Y, Gao X, Zhang M, Yan S, Sun C, Xiao F, Huang N, Yang X, Zhao K, Zhou H, Huang S, Xie B, Zhang N. Novel role of FBXW7 circular RNA in repressing glioma tumorigenesis. J Natl Cancer Inst. 2018. https://doi. org/10.1093/jnci/djx166.

32. Hu W, Bi ZY, Chen ZL, Liu C, Li LL, Zhang F, Zhou Q, Zhu W, Song YY, Zhan BT, Zhang Q, Bi YY, Sun CC, Li DJ. Emerging landscape of circular RNAs in lung cancer. Cancer Lett. 2018;427:18-27.

33. Li, , Yang $X$, Yuan W, Yang $C$, Zhang $X$, Han J, Wang J, Deng $X$, Yang $H$, Li P, Tao J, Lu Q, Gu M. CircRNA-Cdr1as exerts anti-oncogenic functions in bladder cancer by sponging MicroRNA-135a. Cell Physiol Biochem. 2018:46:1606-16.

34. Li P, Chen S, Chen H, Mo X, Li T, Shao Y, Xiao B, Guo J. Using circular RNA as a novel type of biomarker in the screening of gastric cancer. Clin Chim Acta. 2015;444:132-6.

35. Jiang $X M$, Li ZL, Li JL, Xu Y, Leng KM, Cui YF, Sun DJ. A novel prognostic biomarker for cholangiocarcinoma: circRNA Cdr1as. Eur Rev Med Pharmacol Sci. 2018:22:365-71.

36. Xu L, Zhang M, Zheng X, Yi P, Lan C, Xu M. The circular RNA ciRS-7 (Cdr1as) acts as a risk factor of hepatic microvascular invasion in hepatocellular carcinoma. J Cancer Res Clin Oncol. 2017;143:17-27. 
37. Yang $X$, Xiong $Q$, Wu Y, Li S, Ge F. Quantitative proteomics reveals the regulatory networks of circular RNA CDR1as in hepatocellular carcinoma cells. J Proteome Res. 2017;16:3891-902.

38. Zhang Y, Liang W, Zhang P, Chen J, Qian H, Zhang X, et al. Circular RNAs: emerging cancer biomarkers and targets. J Exp Clin Cancer Res. 2017;36:152.

39. Cortes-Lopez M, Miura P. Emerging functions of circular RNAs. Yale J Biol Med. 2016;89:527-37.

40. Lewis BP, Burge CB, Bartel DP. Conserved seed pairing, often flanked by adenosines, indicates that thousands of human genes are microRNA targets. Cell. 2005;120:15-20.

41. Lou K, Chen N, Li Z, Zhang B, Wang X, Chen Y, et al. MicroRNA-142-5p overexpression inhibits cell growth and induces apoptosis by regulating FOXO in hepatocellular carcinoma cells. Oncol Res. 2017;25:65-73.

42. Tsang FH, Au SL, Wei L, Fan DN, Lee JM, Wong CC, et al. MicroRNA142-3p and microRNA-142-5p are downregulated in hepatocellular carcinoma and exhibit synergistic effects on cell motility. Front Med. 2015:9:331-43.

43. Fu L, Balasubramanian M, Shan J, Dudenhausen EE, Kilberg MS. Autoactivation of c-JUN gene by amino acid deprivation of hepatocellular carcinoma cells reveals a novel c-JUN-mediated signaling pathway. J Biol Chem. 2011;286:36724-38.

44. Hsu TY, Fourel G, Etiemble J, Tiollais P, Buendia MA. Integration of hepatitis virus DNA near c-myc in woodchuck hepatocellular carcinoma. Gastroenterol Jpn. 1990;25(Suppl 2):43-8.

45. Ma WL, Jeng LB, Lai HC, Liao PY, Chang C. Androgen receptor enhances cell adhesion and decreases cell migration via modulating beta1integrin-AKT signaling in hepatocellular carcinoma cells. Cancer Lett. 2014:351:64-71.

46. Hishida M, Nomoto S, Inokawa Y, Hayashi M, Kanda M, Okamura Y, et al. Estrogen receptor 1 gene as a tumor suppressor gene in hepatocellular carcinoma detected by triple-combination array analysis. Int J Oncol. 2013:43:88-94.

47. Yang XW, Shen GZ, Cao LQ, Jiang XF, Peng HP, Shen G, et al. MicroRNA-1269 promotes proliferation in human hepatocellular carcinoma via downregulation of FOXO1. BMC Cancer. 2014;14:909.

48. Lei T, Ling X. IGF-1 promotes the growth and metastasis of hepatocellular carcinoma via the inhibition of proteasome-mediated cathepsin B degradation. World J Gastroenterol. 2015;21:10137-49.

49. Paschoal JP, Bernardo V, Canedo NH, Ribeiro OD, Caroli-Bottino A, Pannain VL. Microvascular density of regenerative nodule to small hepatocellular carcinoma by automated analysis using CD105 and CD34 immunoexpression. BMC Cancer. 2014;14:72.
50. zniewski P, Tournigand C. Unravelling the pharmacologic opportunities and future directions for targeted therapies in gastro-intestinal cancers part 2: neuroendocrine tumours, hepatocellular carcinoma, and gastrointestinal stromal tumours. Pharmacol Ther. 2018;181:49-75.

51. Qu XA, Rajpal DK. Applications of connectivity map in drug discovery and development. Drug Discov Today. 2012;17:1289-98.

52. Lamb J. The connectivity map: a new tool for biomedical research. Nat Rev Cancer. 2007;7:54-60.

53. Tao SF, Zhang CS, Guo XL, Xu Y, Zhang SS, Song JR, et al. Anti-tumor effect of 5 -aza-2'-deoxycytidine by inhibiting telomerase activity in hepatocelular carcinoma cells. World J Gastroenterol. 2012;18:2334-43.

54. Xing $T J, X u H T$, Yu WQ, Jiang DF. Methylation regulation of liver-specific microRNA-122 expression and its effects on the proliferation and apoptosis of hepatocellular carcinoma cells. Genet Mol Res. 2013;12:3588-97.

55. Skarn M, Baroy T, Stratford EW, Myklebost O. Epigenetic regulation and functional characterization of microRNA-142 in mesenchymal cells. PLoS ONE. 2013;8:e79231.

56. Villegas SN, Gombos R, Garcia-Lopez L, Gutierrez-Perez I, Garcia-Castillo J, Vallejo DM, et al. PI3K/Akt cooperates with oncogenic notch by inducing nitric oxide-dependent inflammation. Cell Rep. 2018;22:2541-9.

57. Rahman AF, Korashy HM, Kassem MG. Gefitinib. Profiles Drug Subst Excip Relat Methodol. 2014;39:239-64.

58. Gu HR, Park SC, Choi SJ, Lee JC, Kim YC, Han CJ, et al. Combined treatment with silibinin and either sorafenib or gefitinib enhances their growthinhibiting effects in hepatocellular carcinoma cells. Clin Mol Hepatol. 2015;21:49-59.

59. Shao J, Xu Z, Peng X, Chen M, Zhu Y, Xu L, et al. Gefitinib synergizes with irinotecan to suppress hepatocellular carcinoma via antagonizing Rad51mediated DNA-repair. PLoS ONE. 2016;11:e0146968.

60. Rossi L, Zoratto F, Papa A, lodice F, Minozzi M, Frati L, et al. Current approach in the treatment of hepatocellular carcinoma. World J Gastrointest Oncol. 2010;2:348-59.

61. Yu F, Liu JB, Wu ZJ, Xie WT, Zhong XJ, Hou LK, et al. Tumor suppressive microRNA-124a inhibits stemness and enhances gefitinib sensitivity of non-small cell lung cancer cells by targeting ubiquitin-specific protease 14. Cancer Lett. 2018:427:74-84

62. Cao X, Lai S, Hu F, Li G, Wang G, Luo X, et al. miR-19a contributes to gefitinib resistance and epithelial mesenchymal transition in non-small cell lung cancer cells by targeting c-Met. Sci Rep. 2017;7:2939.

63. Wang Z, Pan L, Yu H, Wang Y. The long non-coding RNA SNHG5 regulates gefitinib resistance in lung adenocarcinoma cells by targeting miR-377/ CASP1 axis. Biosci Rep. 2018. https://doi.org/10.1042/BSR20180400.
Ready to submit your research? Choose BMC and benefit from:

- fast, convenient online submission

- thorough peer review by experienced researchers in your field

- rapid publication on acceptance

- support for research data, including large and complex data types

- gold Open Access which fosters wider collaboration and increased citations

- maximum visibility for your research: over $100 \mathrm{M}$ website views per year

At $\mathrm{BMC}$, research is always in progress.

Learn more biomedcentral.com/submissions 